\title{
Evaluating the rubber sheet spacetime analogy by studying ball movement in a bent trampoline
}

\author{
Pau Batlle $^{1}$, Adam Teixidó ${ }^{1}$, Joan Llobera ${ }^{1}$, Isabel Medrano ${ }^{1}$, \\ Luis Carlos Pardo ${ }^{2}$ \\ ${ }^{1}$ Universitat Politècnica de Catalunya, ETSETB, Enginyeria Física, Barcelona, Spain \\ ${ }^{2}$ Grup de Caracterització de Materials, Departament de Física, EEBE, Universitat \\ Politècnica de Catalunya, Barcelona, Spain \\ E-mail: pau.batlle.franch@gmail.com
}

\begin{abstract}
A usual qualitative analogy used to explain gravitation in general relativity is comparing spacetime warping by massive objects with deformation in a rubber sheet. Motivated by this analogy, which identifies planet orbits with trajectories of rolling objects on the rubber sheet, the movement of a small ball in a trampoline bent because of the presence of a heavy mass in its center is studied. It is concluded that the similarities between how masses move under warped spacetime and under a warped trampoline are only qualitative, and later some analogy flaws are outlined, which can be useful for general relativity teaching. Since the "relativistic model" does not match the ball movement in the experimental conditions, two models based on classical mechanics are presented to describe it.

The models are implemented computationally and parameters of such models are optimized to match experimental trajectories. In the case of the most complex of these two models, the high accuracy between optimized and observed trajectories implies that the model is able to explain the experiment behaviour.
\end{abstract}

\section{Introduction}

Emotions are a key factor to enhance students performance in complex subjects such as physics $[1,2]$. A place where the entanglement of emotions and physics laws take place in a natural way are amusement parks. The goal of FISIDABO project [3] is to involve students in measuring and analyzing physical quantities, promoting their engagement in a very simple research project designed by them and performed in Tibidabo, the amusement park of Barcelona. The opportunity to be involved in a more complex project is given to some of those students with high capabilities in physics and mathematics. This part of the more general FISIDABO project, designed for all students, has been given the name of HIPATIA, and aims to give the opportunity to those students to develop 
their own project supervised by university teachers. As an example students have investigated the dynamics of crash cars [4] or the movement of a pendulum in a rotating plane [5]. The present paper presents research triggered by the classical divulgation experiment to explain General Relativity (GR) showing the trajectory of a ball in an elastic sheet bent by a central weight.

One of the most novel concepts Einstein introduced in his theory of GR is the connection between gravitational forces and spacetime geometry. In this theory, gravity is not thought anymore as a force between objects due to its masses. Instead, gravity appears as the result of the mass of an object warping the spacetime around it. Objects follow geodesics when moving in spacetime only under the effects of gravity, the warping alters the geodesics, in turn changing the trajectories. Since spacetime warping can be a difficult concept to understand and visualize, an analogy is often used in physics education: one can think of spacetime as a thin rubber sheet. The way masses warp the rubber sheet and affect other smaller masses can help students developing an intuition about how gravity works under GR.

The objective of this article is to evaluate this analogy by first warping a trampoline by adding weight in its center and then recording small ball trajectories orbiting around the center.

\section{Experimental methods}

The two main goals of the experiment were, on the one hand, warping the trampoline and measuring its shape, and on the other hand, recording the movement of a small ball orbit around another, bigger ball, placed in the center.

\subsection{Warping the trampoline and recording its shape}

An illustration of the experimental design including the central ball along with the trampoline measures can be found in Figure 1.

The ball placed in the center of the trampoline was a bowling ball with a mass of 7.26 $\mathrm{kg}$ and a radius of $10.6 \mathrm{~cm}$, playing the role of a central mass or a star for planets to orbit around. Since the mass from the ball itself did not suffice to warp the trampoline enough for our purposes, lifting weights with a combined mass of $60 \mathrm{~kg}$ were attached to the trampoline in the center of the ball to increase the overall deformation of the trampoline, as seen in Figure 2. This figure is part of a video which records a lateral view of the trampoline that was later used for reconstruction of the trampoline shape, as explained in the section raw data analysis. 


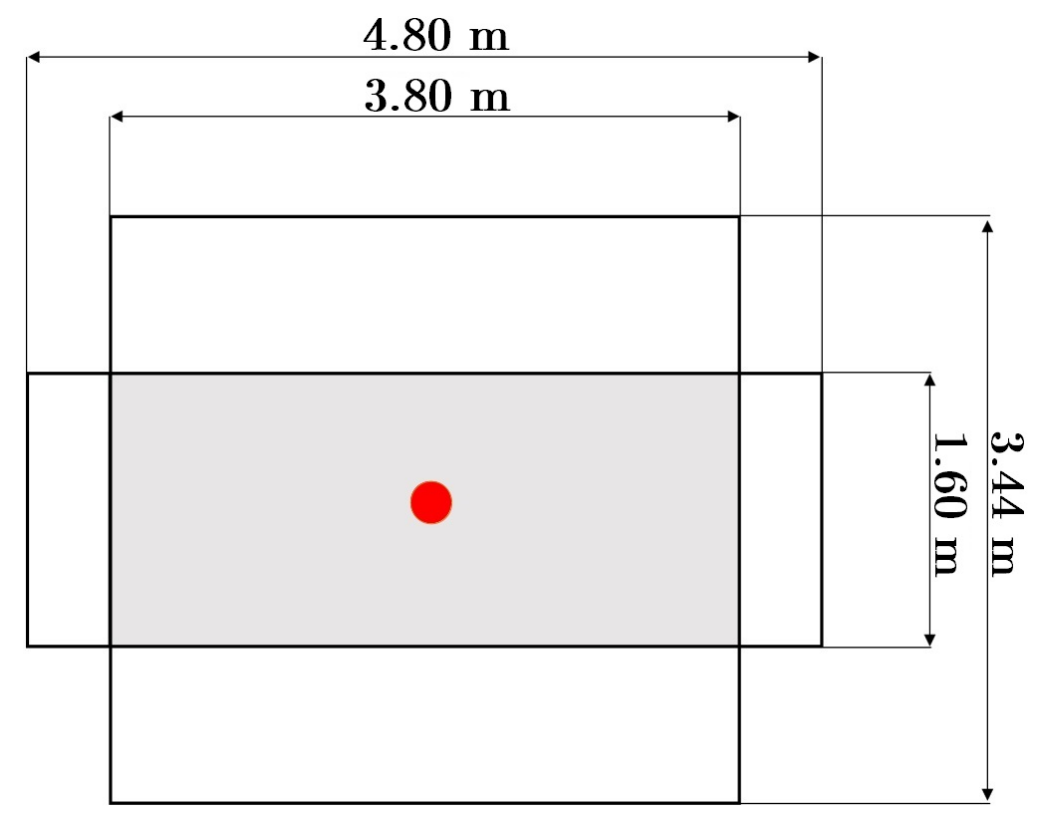

Figure 1: This figure shows the measures (in $m$ ) of the trampoline and adjacent platforms used in the experiment together with the position of the central ball

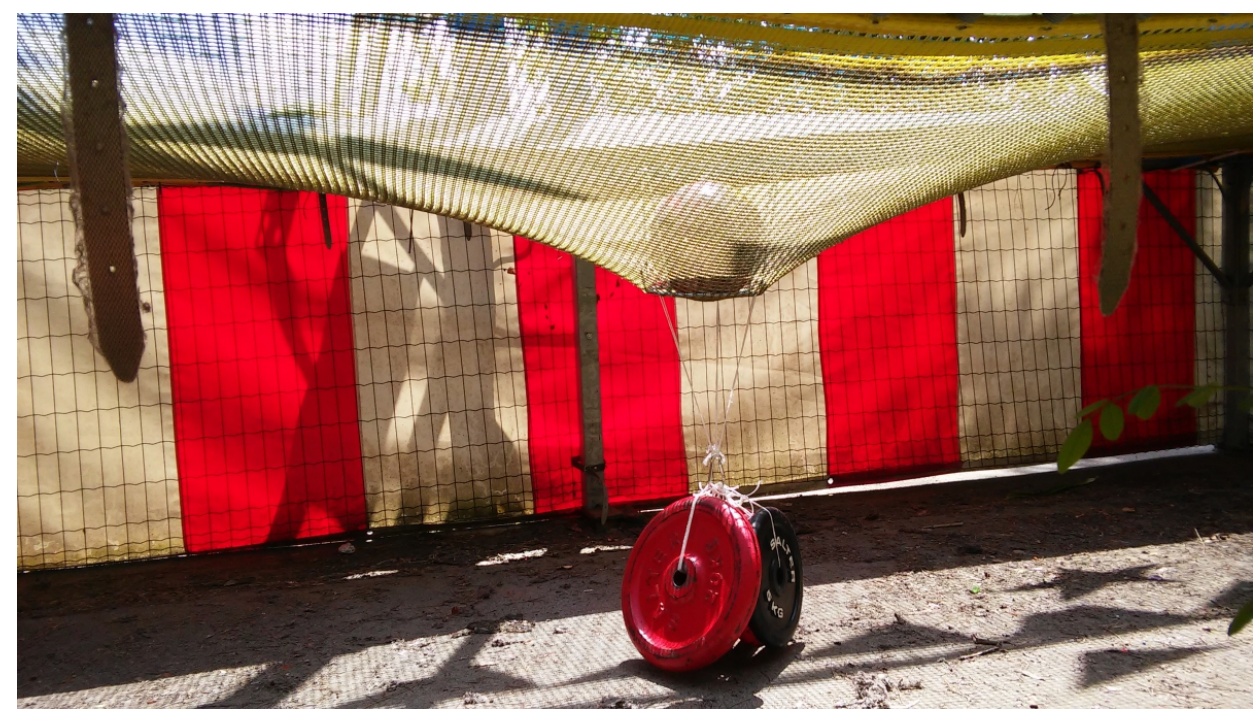

Figure 2: Lateral view of the warped elastic bed due to the total mass of $67.3 \mathrm{~kg}$. It was made sure that the lifting weights did not touch the floor

\subsection{Recording the movement of the ball}

The small ball, playing the role of a planet orbiting a star, was a petanque ball with a radius of $3.52 \mathrm{~cm}$ and a mass of $0.718 \mathrm{~kg}$. Different throws of the small ball on the surface of the trampoline were performed. The ball was ejected with an angle and a velocity such that it 'orbited' around the central mass for more than 5 seconds, which was useful to record the movement allowing for the posterior analysis of the trajectory 
of the ball.

The experiments were recorded with a video camera located over the central mass position and an approximate height of 1 meter. A video take was recorded for each different ball throw. The camera used had a wide angle objective and no deviation between the images (frames) taken by the camera and the real position of the ball was assumed. The videos were recorded at $180 \mathrm{fps}$ (frames per second). An example of a frame of the videos captured by the camera can be seen in Figure 3 .

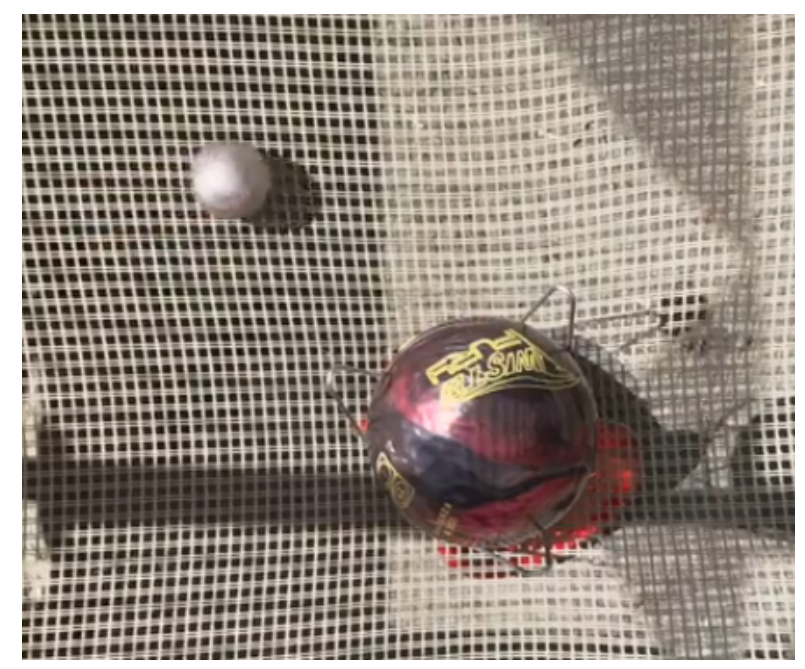

Figure 3: Example of a frame obtained from one of the ball throws. Both balls used in the experiment can be seen

\section{Raw Data Analysis}

Using the raw data obtained during the experiment, both the filmed 2D trajectories of the small ball and an approximation for the shape of the elastic bed were found. One should note that both things are necessary to fully reconstruct the experiment and for later physical analysis: the trajectory of the rolling ball is filmed from above, only giving information of its position $(x, y)$ on the horizontal plane with respect to the central ball. The shape of the trampoline completes the position of the petanque ball in $3 \mathrm{D}$ by providing the altitude at which the ball must be at every $(x, y)$ point.

\subsection{Obtaining the trajectories}

Given the videos recorded at $180 \mathrm{fps}$, the position data was extracted by analyzing the video frame by frame using the program Tracker Video Analysis and Modeling Tool. [6] The program was used to manually track the trajectory of the center of the small ball 
with respect to the middle of the central mass, which acts as our coordinate origin (since its movement is almost nonexistent, and therefore can be ignored). After exporting the position data, other magnitudes such as velocity, acceleration and angular magnitudes such as distance from the centre and swept angle of the ball were calculated for each take at each frame. All the data was smoothed using a Savitzky-Golay filter for convenience in later use. The trajectories observed are shown in Figure 4.
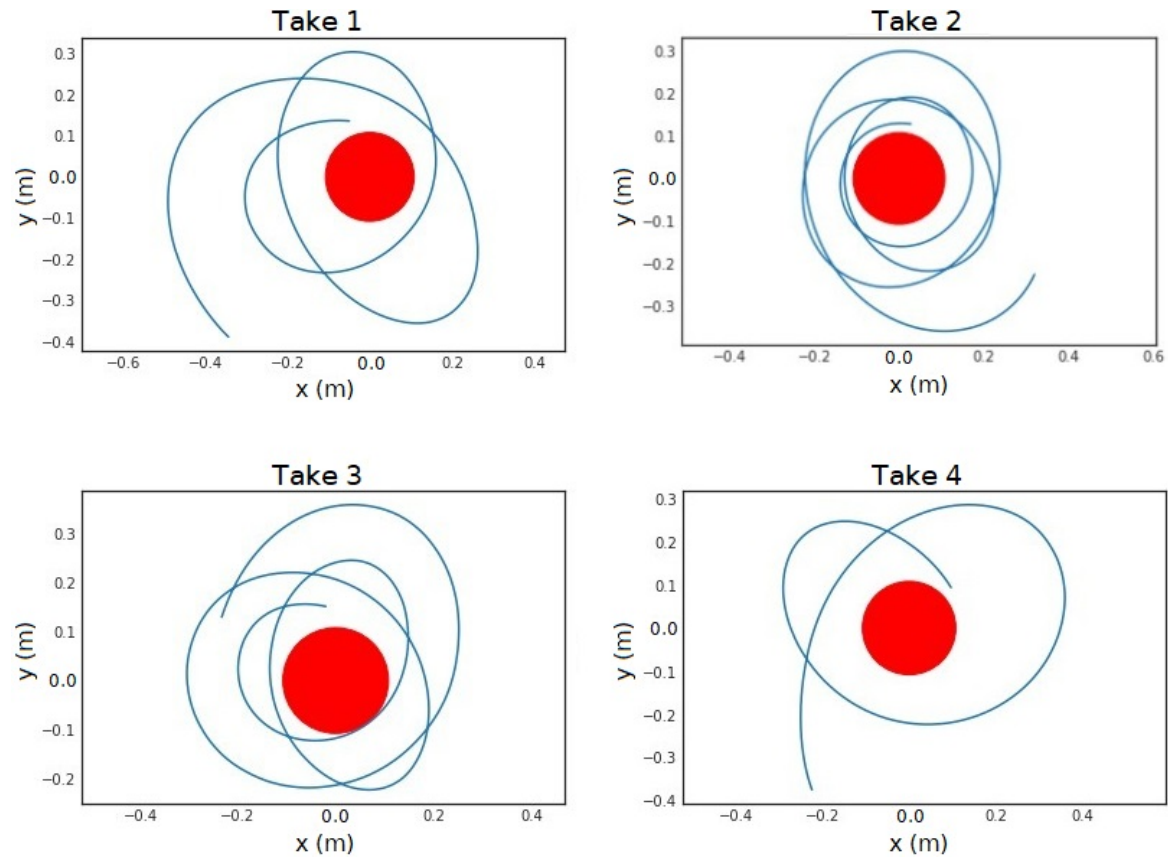

Figure 4: Smoothed trajectories of the petanque ball obtained after video analysis

\subsection{Measuring the shape of the trampoline}

To recover the height of the trampoline, an image tool that allows to measure the positions of specific points is used (see Figure 5). Previously, the image perspective was corrected using an image editor, in order to make the trampoline longer axis parallel to the photo's left to right direction. The coordinate system used along this article has its origin in the center of the bowling ball, $x$-axis and $y$-axis are parallel to the longer and the shorter axis of the trampoline respectively and the z-axis is the vertical one. Therefore, in the image of Figure 5, the x-axis goes from left to right and the z-axis goes from bottom to top.

Finally, heights and $x$-axis positions in meters can be recovered from the image measurements using a reference object with a known length, such as the diameter of the bowling ball. The coordinates of the measured surface points in $x$-axis and $z$-axis are refered as $(x, z)$.

In order to have a simple expression for the height of the trampoline, a function $z=h(x)$ 


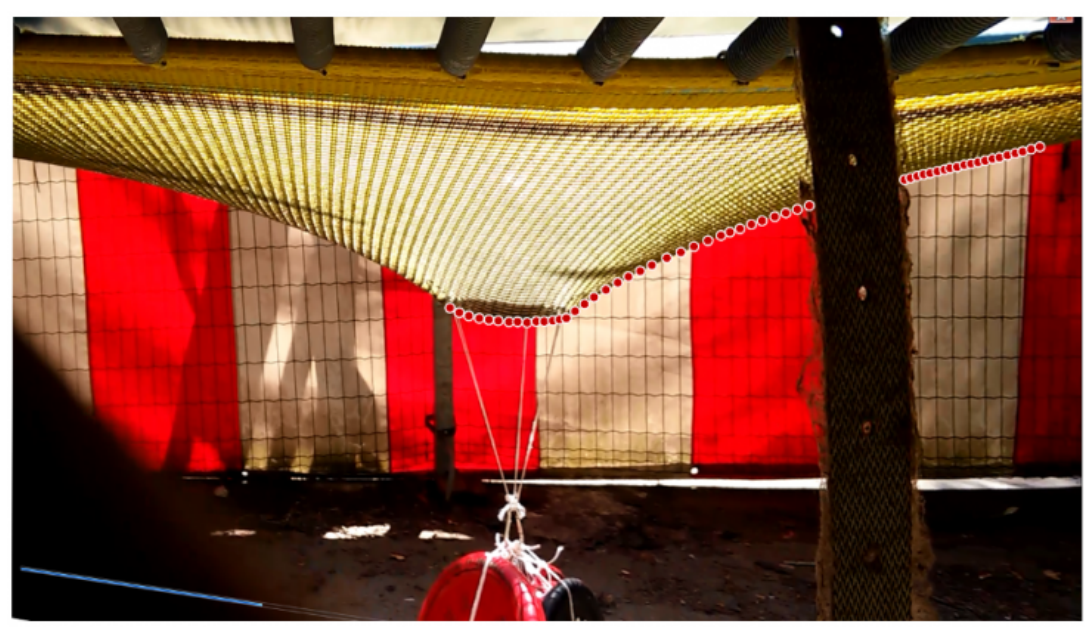

Figure 5: Perspective corrected trampoline. Red dots are the collected position points

is fitted to the surface experimentally recovered points. The $h$ function found to both have a simple expression and minimize the mean square error respect to the measured heights has the form:

$$
h(x)=-k e^{-c x} \quad \text { with } k, c>0
$$

Where by minimizing the distance between all measured $z$ coordinates and their corresponding $h(x)$, the parameters are estimated to be $k=0.44 \mathrm{~m}$ and $c=1.8 \mathrm{~m}^{-1}$.

This function only provides the height along the $x$-axis. A first step to reconstruct the full trampoline's shape would be to consider that it has radial symmetry. Then the expression for the complete height $f$ would be $f(x, y)=h\left(\sqrt{x^{2}+y^{2}}\right)$. However, since the trampoline is not circular, elliptic symmetry is more sensible. The final expression for the height $f$ becomes:

$$
\begin{array}{r}
f(x, y)=h(r(x, y))=-k e^{-c r(x, y)} \\
\text { where } r(x, y)=\sqrt{x^{2}+(\sigma y)^{2}}
\end{array}
$$

With $\sigma$ being a free parameter referred to as the "stretching parameter". $\sigma$ only stretches in the $y$-axis in order to recover that the height is $h(x)$ when $y=0$. This fitted exponential function serves as a first, simple approximation for the trampoline shape. Another method to approximate the shape which does not require the former symmetry assumption nor the stretching parameter will be developed in the modelling section.

\section{Evaluating the spacetime rubber sheet analogy}

As discussed previously, the spacetime rubber sheet analogy can help developing an intuition about spacetime curvature. However, in this section it will be seen that the analogy is limited since it can not be extended to some facets. Two tests were performed: a comparison between the trajectories observed and the ones that GR postulates would 
predict if the trampoline played the complete role of spacetime, and the comparison between the apsidal precession of the orbits observed and the predicted by GR for orbits of planets in the solar system.

\subsection{Trajectory shape comparison}

Since trajectories in spacetime are known to be geodesics, and considering the trampoline to play the complete role of spacetime in the analogy analized, a sensible first approach is analizing the trampoline geodesics. Geodesics are the curves which minimize distance between two points, and it can be proven mathematically that geodesics in surfaces correspond to trajectories followed by particles only under forces which are perpendicular to the surface [7]. Therefore, the geodesics of the surface were calculated by simulating the path of a particle only under the normal force of the surface, which is perpendicular to the surface itself. This can be achieved using the Algorithm 2 described in the trajectory simulation section, setting all forces but the normal force to zero. As it can be observed in Figure 6, due to the trampoline shape, its geodesics are always "open", this is, no orbits can be observed. A bounded orbit would not satisfy the property of forces being perpendicular to the surface at all points. This however does not match the observed trajectories, shown in Figure 4. Instead of following trampoline geodesics, orbits are formed thanks to Earth gravity acting on the petanque ball and making it stay trapped on the trampoline deformation. Also, the orbits gradually reduce in size due to friction. Since the trampoline geodesics do not correspond to ball trajectories over the surface, the analogy cannot be applied in this manner. Assuming that particles should follow the trampoline geodesics in the conditions of this experiment is an incorrect extrapolation of the rubber sheet analogy.

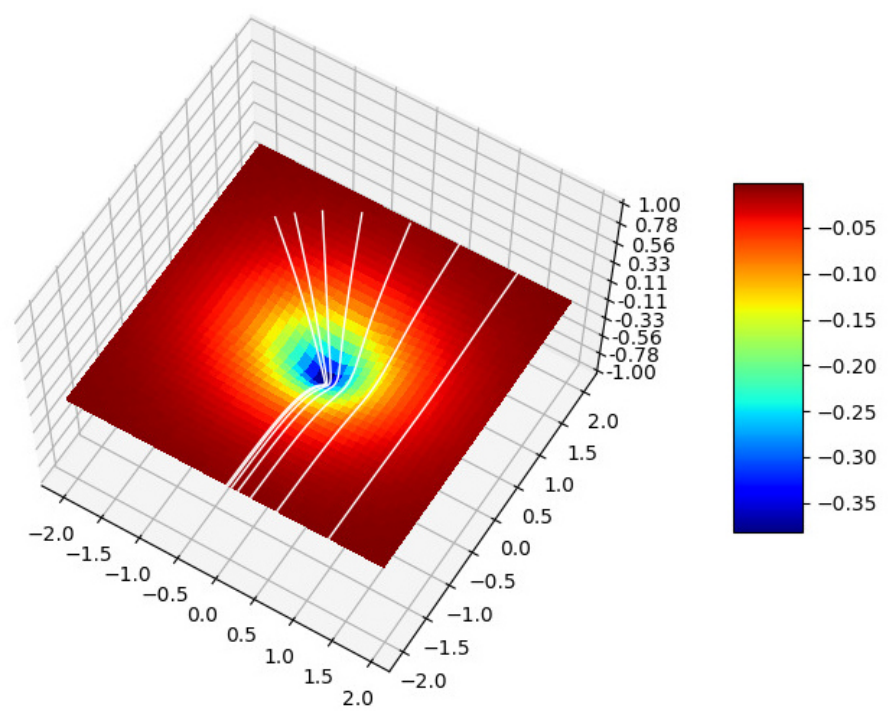

Figure 6: Various trampoline geodesics 


\subsection{Apsidal precession comparison}

As noted in the previous subsection, trajectories around the central ball do not follow the trampoline geodesics but orbit the center instead. It is precisely because of this fact that the analogy can be used in the first place: the visual resemblance between the ball movement and planet orbits can help students visualizing the effects of spacetime warping.

To study this resemblance, a comparison between the observed orbits and predicted GR orbits such as the ones observed in the solar system is made. In particular, classical orbital parameters such as the period of revolution, semi-major axis, orbit eccentricity and apsidal or perihelion precession are calculated, to later discuss whether these parameters obey the relation predicted by GR, given by

$$
\sigma_{p e r i} \propto \frac{L^{2}}{T^{2}\left(1-e^{2}\right)}
$$

where we observe the dependence of the perihelion precession $\sigma_{\text {peri }}$ on the parameters $L$ (semi-major axis), $T$ (period of revolution) and $e$ (eccentricity of the orbit). Historically, the correct prediction of the apsidal precession of Mercury given by the expression above is considered a major proof of validity of the GR theory, since previous gravitational theories failed to completely explain why and how Mercury's perihelion precessed over time.

The study of the distance between centers $r$ (orbital radius) as a function of time $t$ can be used to obtain the orbital data. The $r(t)$ plots of takes 1 to 4 can be found in Figure 7 , and Figure 8 represents some of the parameters we want to obtain.
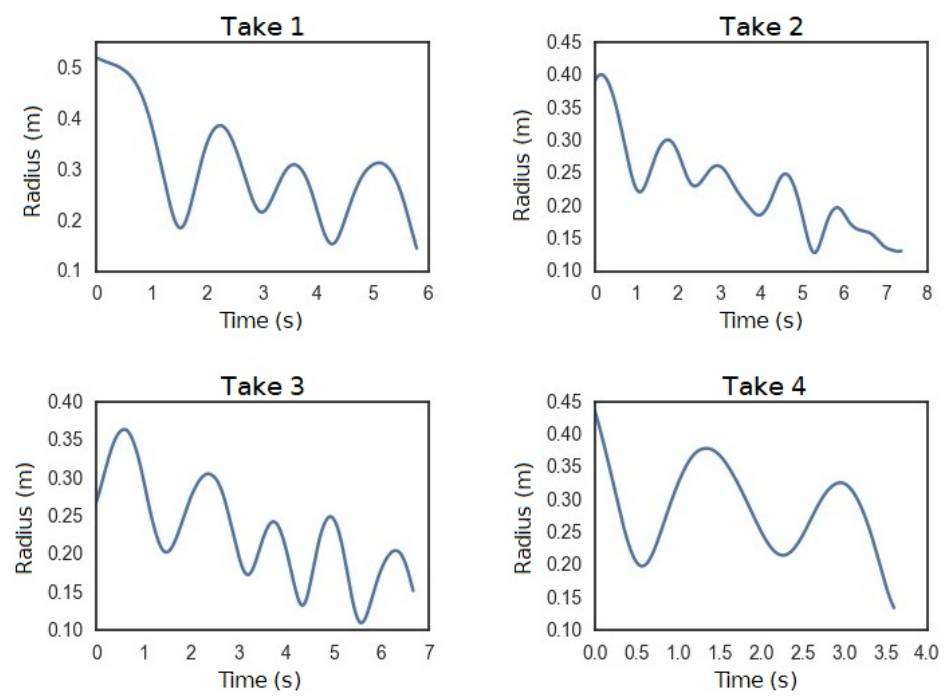

Figure 7: Graphical representation of $r$ as a function of $t$, of takes 1 to 4 


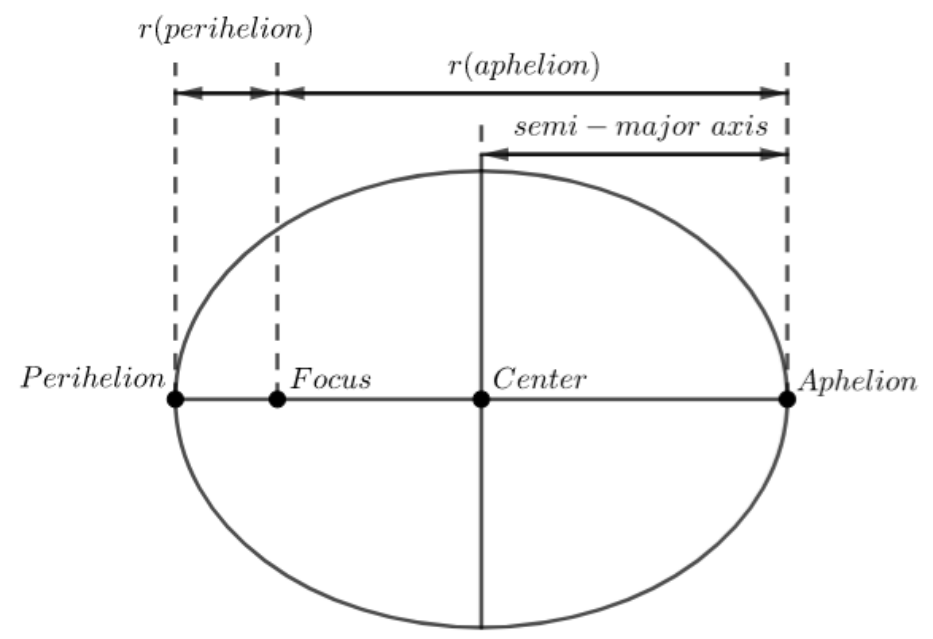

Figure 8: Some parameters of the ellipse

In order to obtain the parameters from the data, it is considered for each orbit that the interval between two consecutive maximum values of $r(t)$ are separate "ellipses". As in planetary orbits, we consider the bowling ball to be placed in a focus of the ellipse. Also, since friction causes orbital radius to decay over time, orbital parameters will slowly change while the ball is following the ellipse.

Let $t_{\min }$ be the value in this interval where the radius is minimum and $t_{\max 1}$ and $t_{\max 2}$ be the two extremes of the interval (where $r(t)$ attains a local maxima). Two more time values for each ellipse are needed for the calculation: the two instants, $t_{m i d 1}$ and $t_{m i d 2}$, at which the ball is crossing the minor axis. An estimation of $t_{m i d 1}$ and $t_{m i d 2}$ is obtained by considering the crossing to happen when $1 / 4$ or $3 / 4$ of the length is travelled from the aphelion, analogously to a real ellipse. The orbital parameters are calculated as explained on Table 1.

\begin{tabular}{ll} 
Orbital parameter & Calculation Method \\
\hline Period of revolution $(T)$ & $t_{\text {max } 2}-t_{\max 1}$ \\
$r$ at Perihelion $\left(r_{\text {perihelion }}\right)$ & $r\left(t_{\text {min }}\right)$ \\
$r$ at Aphelion $\left(r_{\text {aphelion }}\right)$ & $\left(r\left(t_{\text {max } 1}\right)+r\left(t_{\text {max } 2}\right)\right) / 2$ \\
$r(t)$ at minor axis $\left(r_{\text {mid }}\right)$ & $\left(r\left(t_{\text {mid } 1}\right)+r\left(t_{\text {mid } 2}\right)\right) / 2$ \\
Semi-major axis $(L)$ & $\left(r_{\text {aphelion }}+r_{\text {perihelion }}\right) / 2$ \\
Semi-minor axis $(l)$ & $\sqrt{r_{\text {mid }}^{2}-\left(L-r_{\text {perihelion }}\right)^{2}}$ \\
Eccentricity & $\sqrt{1-\frac{l^{2}}{L^{2}}}$ \\
Apsidal precession & $\mid$ Angle diff between maxima $\mid-2 \pi$
\end{tabular}

Table 1: Calculation methods used for different classical orbital parameters

The results of the ten analyzed orbit fragments or ellipses can be found in Table 2. Only their mean and standard deviation are presented because their variation is relatively 
small.

\begin{tabular}{ccc} 
Orbital parameter & Mean value observed & Standard deviation \\
\hline Semi-major axis $(L)$ & $0.24 \mathrm{~m}$ & $0.04 \mathrm{~m}$ \\
Semi-minor axis $(l)$ & $0.23 \mathrm{~m}$ & $0.04 \mathrm{~m}$ \\
Period of revolution $(T)$ & $1.4 \mathrm{~s}$ & $0.2 \mathrm{~s}$ \\
Eccentricity $(e)$ & 0.29 & 0.07 \\
Apsidal precession $\left(\sigma_{\text {peri }}\right)$ & $-2.1 \mathrm{rad}$ & $0.5 \mathrm{rad}$
\end{tabular}

Table 2: Results of the calculated orbital parameters

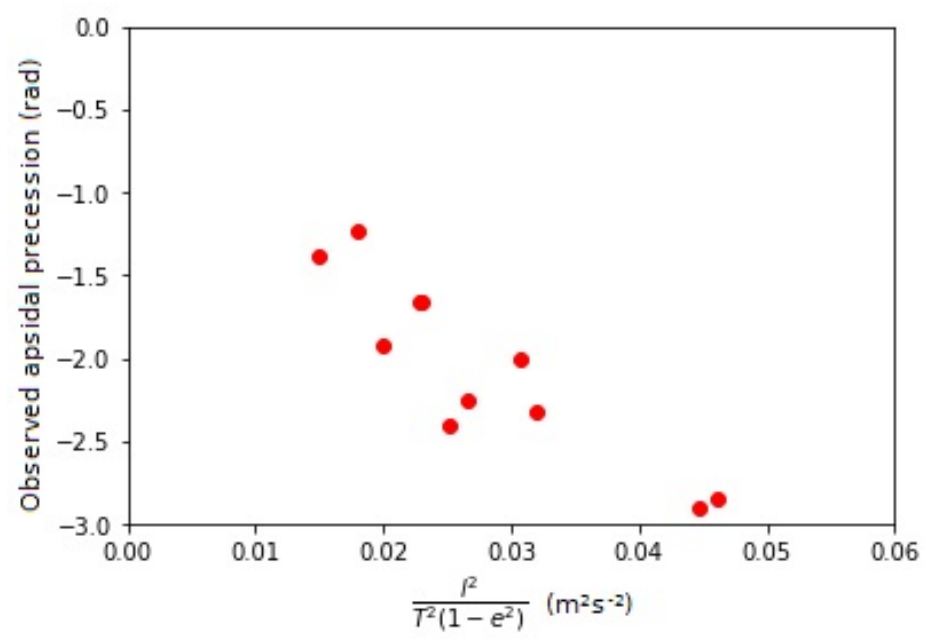

Figure 9: Relationship between apsidal precession and orbital parameters

The first interesting result is that all the apsidal precession values found are negative. Oppositely, GR predicts them to be positive. In other words, unlike what happens in planet orbits maxima in radial distance repeat before a $2 \pi$ radian revolution is completed.

More concisely, the GR relation predicts that in Figure 9 plot, a linear relation with positive slope should be observed, which is clearly not the case of the obtained experimental data.

In conclusion, the analogy is flawed when it is taken into the context of analyzing the orbit, as the GR prediction about the relation between the studied orbital parameters has been found to not apply to the ball orbits in the trampoline. This conclusion coincides with the results obtained by C.A. Middleton and M. Langston in [8]. They prove that no Newtonian-like orbits (excluding circular orbits) will appear on any cylindrically-symmetric surface, and the same inconsistency holds for orbits obtained by a full GR treatment. 
The results of this section indicate that taking the analogy one step further, by not only using it to get familiarized with spacetime curvature but also using it to predict properties about the orbits on the rubber sheet, does not work. Also, the consideration stated in the first comparison of this section, the rubber sheet being spacetime, is not the most accurate way to present the analogy. Instead, the sheet being a slice of space, deformed by masses, is an excellent analogy and a visualisation of space warped by a black hole, as it is pointed out by K. Thorne in his published lecture notes [9].

\section{Physical Models}

Having concluded in the previous section that the rubber sheet spacetime analogy does not match the movement of the orbiting ball in the experimental conditions, a physical model to explain the observed trajectories is lacking. In this section, a model of the trampoline shape and two models of the ball movement under the influence of the bent trampoline are presented. These three models are based solely on classical mechanics.

In both physical models of the ball movement presented, specifying the surface where the ball moves is required. With the purpose of providing it to the model, the trampoline surface parametrization can be obtained both as the result of the model presented in this section or the approximation by a radial exponential developed previously.

\subsection{Trampoline shape model}

The trampoline of this experiment can be modeled as an ideally elastic surface, where a known mass has been left on its middle and its rectangular boundary (see Figure 1) has been fixed in place.

To be able to simulate approximately the mentioned scenario, the surface is discretized into a grid of points. Newton's Laws are applied to the grid points, which have a really small mass (coming from the trampoline's elastic cords) together with a great mass distributed in a circle in the center, representing the bowling ball and the weights. These points are united with its neighbours by springs of elastic modulus $K$ which are under a certain initial tension $T_{0}$ when the trampoline is flat, not deformed by the central mass. Both $T_{0}$ and $K$ are not known a priori, and in the following section a method to adjust them computationally will be explained. The equations developed from the stated assumptions and their complete expressions can be found on the annex. As an abridgment, the forces that act on a point with mass $\Delta m$ are tensions $T$ and gravity, which add up to a total force $\vec{F}$,

$$
\vec{F}=\vec{T}_{y}^{-}+\vec{T}_{y}^{+}+\vec{T}_{x}^{-}+\vec{T}_{x}^{+}-\Delta m g \hat{z}
$$


(tensions depend on the four neighbours' positons and $g=9.81 \mathrm{~ms}^{-2}$ is the gravitational acceleration) then the equation of motion for a point in position $\vec{r}$ is

$$
\ddot{\vec{r}}=\frac{\vec{F}}{\Delta m}-\mu \dot{\vec{r}}
$$

where the dot indicates a time derivative, following Newton's notation. A friction term $\mu$ is added to the usual Newton's Law, because in the real experiment friction occurs in and between elastic cords, which dampens oscillations of the surface of the trampoline.

The goal is to determine the shape of the weight-loaded trampoline at equilibrium, when it remains static. Two forces give shape to the trampoline: gravitational forces and elastic forces. At equilibrium these forces compensate each other. As explained in the following section, when implemented computationally, the system is simulated until these equilibrium conditions are reached, using numerical methods to solve the differential equations of the model.

\subsection{Point particle model}

The first physical ball movement model consists of the following assumptions:

(i) The petanque ball is a point particle of mass $m$, without any moment of inertia or radius.

(ii) The point particle can only move over the surface of the trampoline. This happens because the ball is affected by gravity, which obliges it to always touch the elastic trampoline, as it could be seen on the filming of the experiment.

(iii) The trampoline isn't deformed by the passage of the petanque ball, for the sake of simplicity it is considered to be a rigid surface.

(iv) A friction proportional to the normal force is exerted against the particle movement.

Model equations The equations of motion for the punctual particle are immediately obtained from the force $\vec{F}$ exerted on it,

$$
\vec{F}=-m g \hat{z}+\vec{F}_{f}+\vec{F}_{N}
$$

Both friction $\vec{F}_{f}$ and normal $\vec{F}_{N}$ forces are generated by the trampoline,

$$
\begin{gathered}
\vec{F}_{f}=-\mu F_{N} \hat{v} \\
\vec{F}_{N}=\frac{m}{(\vec{\nabla} f)^{2}+1}\left(g+\left[\begin{array}{ll}
\dot{x} & \dot{y}
\end{array}\right] H f\left(\vec{r}_{p}\right)\left[\begin{array}{c}
\dot{x} \\
\dot{y}
\end{array}\right]\right)\left[\begin{array}{ll}
-\vec{\nabla} f & 1
\end{array}\right]
\end{gathered}
$$


Where $F_{N}$ stands for modulus of $\overrightarrow{F_{N}}$, $\hat{v}$ for unitary velocity vector, $\vec{\nabla} f$ and $H f$ for gradient and Hessian of the surface parametrization $f(x, y)$. The positions of the particle in the $x$-axis \& y-axis are $x \& y$ respectively. The derivation of this equations is found on the annex.

Model parameters The calculation of the forces and the model trajectory prediction depend on the parameters listed on Table 3. Note that the mass of the particle $m$ does not appear in the table because its value does not affect the accelerations calculated by the model.

\begin{tabular}{|l|l|l|}
\hline Parameter & Description & Known value \\
\hline$\mu$ & Friction against velocity & No \\
\hline$f(x, y)$ & Parametrized trampoline shape & Estimated \\
\hline$[x, y](0)$ & Initial position of the particle & Yes \\
\hline$[\dot{x}, \dot{y}](0)$ & Initial velocity of the particle & Yes \\
\hline
\end{tabular}

Table 3: Point particle model parameters

\subsection{Rolling sphere model}

The second ball movement model is an improvement of the previous one while still relying on classical Newtonian mechanics. The assumption (i), the petanque ball being a point particle, is dropped, and instead it is considered to be a rigid uniform sphere of radius $R$. Superseding it a new assumption is made:

(i') The sphere rolls on the surface of the elastic trampoline without sliding.

The assumption (ii) has to be more detailed due to the ball now occupying a volume:

(ii') There is always a point $P$ of the sphere surface that is in contact with the trampoline, which is not the same point as the center of masses $O$ of the sphere. This is illustrated in Figure 10.

The assumption (iii) stays the same, the elastic trampoline is not deformed due to the petanque ball's weight. The assumption (iv) is extended by adding a friction torque which diminishes the sphere's spinning.

Model equations Let $\vec{r}_{O}$ and $\vec{r}_{P}=\left[\begin{array}{lll}x_{P} & y_{P} & z_{P}\end{array}\right]$ be the positions of $O$ and $P$ respectively. If the surface of the trampoline is parametrized as $f(x, y)$, then $\vec{n}\left(\vec{r}_{P}\right)$ $=\left[-\vec{\nabla} f\left(x_{P}, y_{P}\right) 1\right]$ is a vector normal to it at point $\mathrm{P}$, the corresponding unitary normal vector is $\hat{n}$. Then, let $\omega_{s}$ be $\vec{\omega} \cdot \hat{n}$, the normal component of the sphere's angular velocity, named spin. 


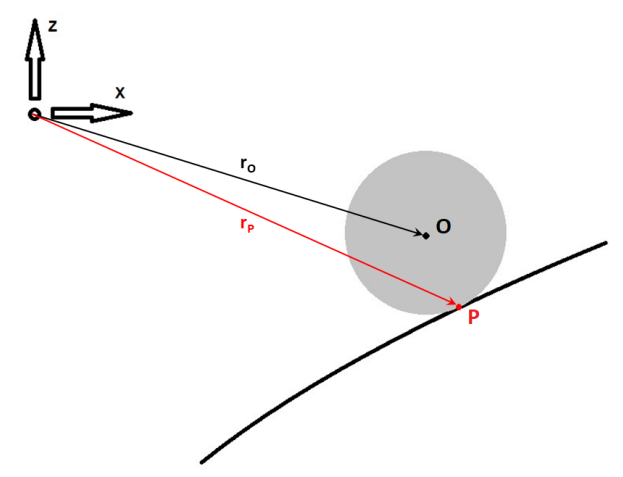

Figure 10: 2D slice of the sphere rolling over the trampoline. The thick black line represents the trampoline surface, and the left top axis marks the coordinate origin

The defined variables $\vec{r}_{O}, \vec{r}_{P}$ and $\omega_{s}$ are unknown, however their first or second derivatives can be expressed as:

$$
\left\{\begin{array}{l}
\ddot{\vec{r}}_{O}=-\left(\dot{\vec{r}}_{O} \cdot \dot{\hat{n}}\right) \hat{n}+\frac{5}{7}\left(\vec{F}_{s}+\vec{F}_{f}-g(\hat{z}-(\hat{z} \cdot \hat{n}) \hat{n})\right) \\
\dot{\vec{r}}_{P}=(I d+R A)^{-1} \dot{\vec{r}}_{O} \\
R \dot{\omega}_{s}=-\dot{\vec{r}}_{O} \cdot(\hat{n} \times \dot{\hat{n}})-\operatorname{sign}\left(\omega_{s}\right) \eta F_{N}
\end{array}\right.
$$

Where:

$$
\begin{aligned}
& \vec{F}_{s}=\frac{2}{5} R \omega_{s} \hat{n} \times \dot{\hat{n}} \\
& \vec{F}_{f}=-C_{r r} F_{N} \hat{v} \\
& F_{N}=m \frac{g}{n}-\dot{\vec{r}}_{O} \cdot \dot{\hat{n}} \\
& \dot{\hat{n}}=A \dot{\overrightarrow{r_{P}}}
\end{aligned}
$$

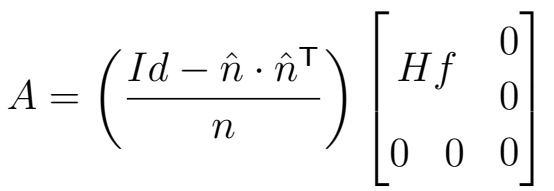

Here $\eta$ is the spin friction coefficient, $C_{r r}$ is the rolling resistance coefficient and $H f$ is evaluated at $\vec{r}_{p}$. This defines a system of ordinary differential equations which is integrated to obtain the sphere trajectory. Again, the full derivation of these equations can be found in the annex.

Model parameters The forces and trajectories predicted by the spherical model depend on the parameters listed on Table 4. As in the point particle case, the sphere mass $m$ does not appear in the table because its value does not affect the calculated accelerations. Also, the initial position of the contact point $\vec{r}_{P}(0)$ does not appear because it is found 
as the solution of the following non-linear equation (which is solved numerically when implementing the model computationally):

$$
\vec{r}_{O}=\vec{r}_{P}+R \hat{n}\left(\vec{r}_{P}\right)
$$

\begin{tabular}{|l|l|l|}
\hline Parameter & Description & Known value \\
\hline$C_{r r}$ & Resistance against rolling coefficient & No \\
\hline$\eta$ & Friction against spin coefficient & No \\
\hline$\omega_{s}(0)$ & Initial value of the ball's spin & No \\
\hline$f(x, y)$ & Trampoline's surface shape & Estimated \\
\hline$\vec{r}_{O}(0)$ & Initial position of the ball's center & Yes \\
\hline$\dot{\vec{r}}_{O}(0)$ & Initial velocity of the ball's center & Yes \\
\hline$R$ & Ball's radius & Yes \\
\hline
\end{tabular}

Table 4: Rolling sphere model parameters

\subsection{Parameter fitting and Model Validation}

The previous section proposed physical models for the trampoline shape and ball movement, which included parameters with unknown values. In order to both validate these models and to find an approximation for the unknown values, computational simulations were performed. In this section the implementation of each of the physical models used to validate them is explained. These simulations were combined together in order to simulate the full experiment, this is, simulating first the shape of the trampoline after adding weight to its center and using the result to simulate the trajectory of a ball on the surface obtained. A diagram of the structure of the combined simulations can be found in Figure 11. This diagram refers to the simulations done using the simulated trampoline via the trampoline model exposed in the previous subsection 5.1, but it should be noted that the exponential fitting derived before (in subsection 3.2) can also be used instead. 


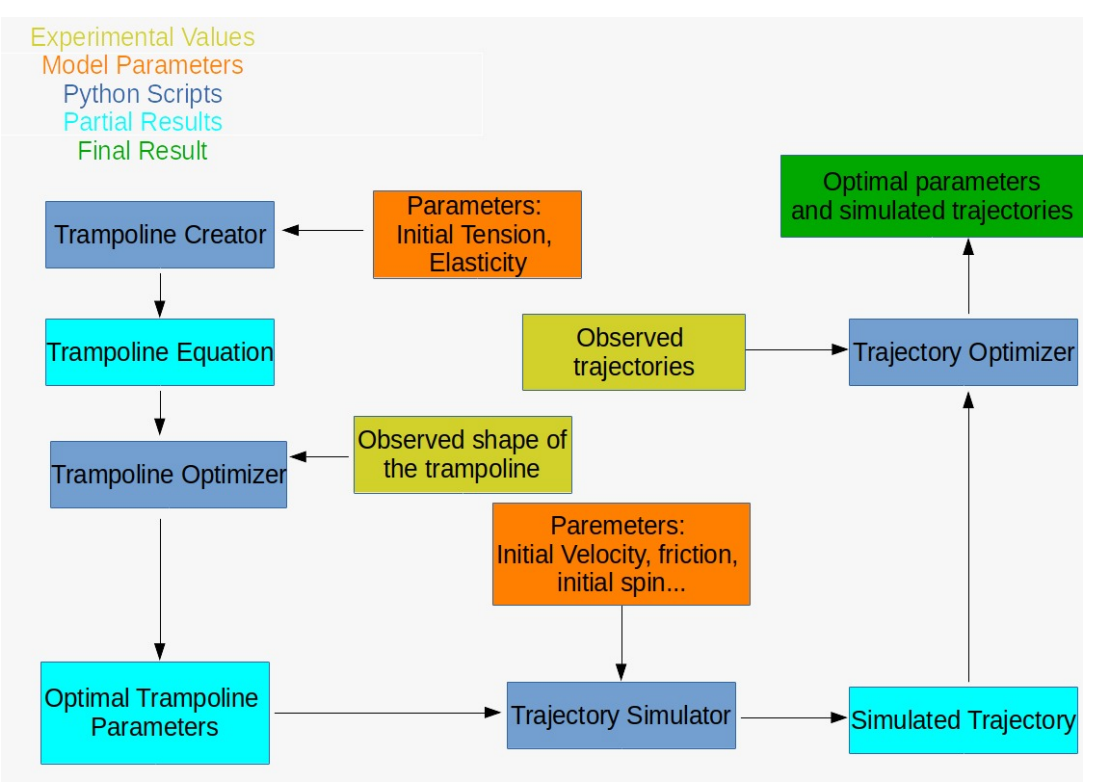

Figure 11: Program flow diagram

5.4.1. Simulating the trampoline shape Given some values for $K$ and $T_{0}$, Algorithm 1 was used to obtain a $3-\mathrm{D}$ reconstruction of the trampoline:

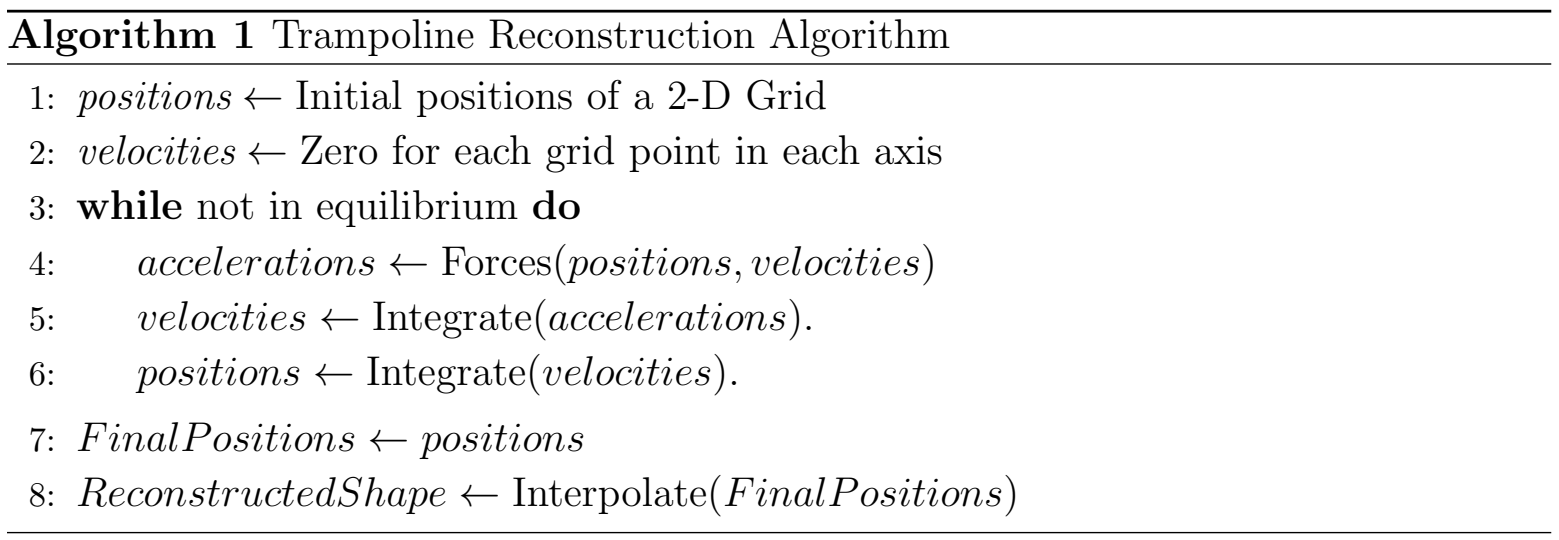

where Forces refers to applying equation 5 from the trampoline shape model and Newton's Law to obtain the accelerations at step $t$ given the positions and velocities at the same step $t$, Integrate refers to applying a numerical integration method to solve the differential equation to obtain the position and velocities at $t+\Delta t$ given the accelerations at $t$ and Interpolate refers to applying an interpolation method in order to be able to know the shape of the trampoline in all points given the final 3-D positions of all the points in the grid. The presence of a friction term in the equations implies that equilibrium will be eventually reached. In this case the specific integration and interpolation numerical methods used were fourth order Runge-Kutta and third order splines, respectively. 
Note that in order to calculate the forces, the values of $K$ and $T_{0}$ need to be specified, and therefore, the final shape of the trampoline depend on their values. The objective is to obtain the ones that generate the most similar trampoline in the $x$-axis to the measurements of the real Tibidabo weight-loaded trampoline. After finding the bestfit values for these two parameters, the predicted trampoline can be used as a good estimation of the real one at every point in the horizontal plane, not only in the $x$-axis. The optimal values for the parameters are found to be $K=112.31 \mathrm{~N}$ and $T_{0}=3.28 \mathrm{~N}$, which generate the trampoline in Figure 12. From this point, $K$ and $T_{0}$ will always be considered to take the optimal values obtained.

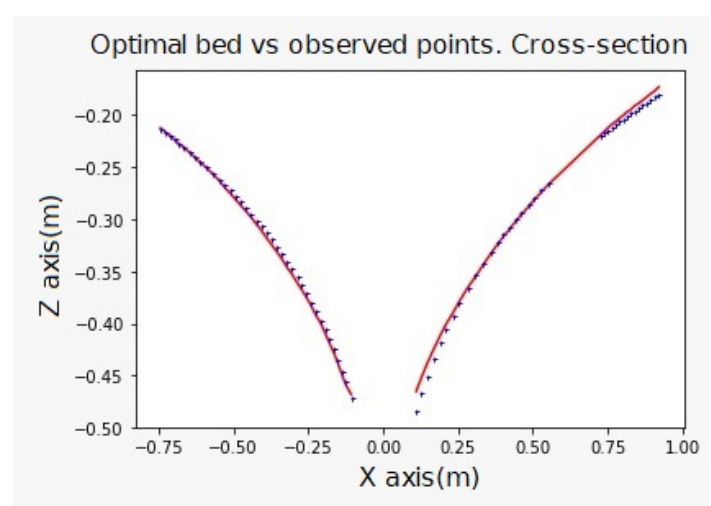

(a) Trampoline (red) and real points (blue)
3D visualization of the optimal trampoline

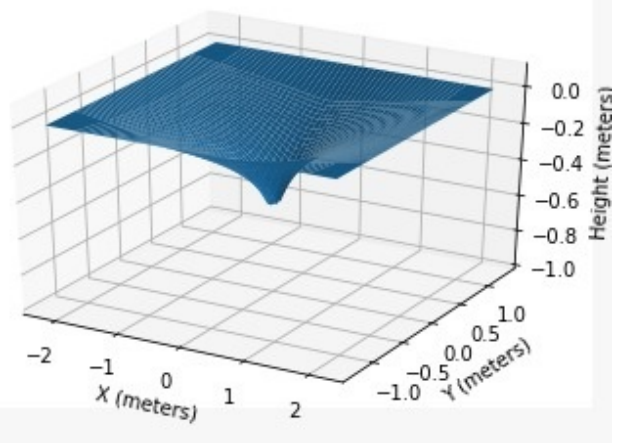

(b) Visualization of the optimization result

Figure 12: Two different views of the simulated trampoline which is most similar to the real one in the $x$-axis cross-section

5.4.2. Simulating the trajectories For both the point particle model and the rolling sphere model, ball trajectories can be simulated using the Algorithm 2. Unlike the previous Trampoline Reconstruction algorithm, in order to obtain the full trajectory information the output has to be the positions, velocities and accelerations of the ball 
at each step, and not only in the last step.

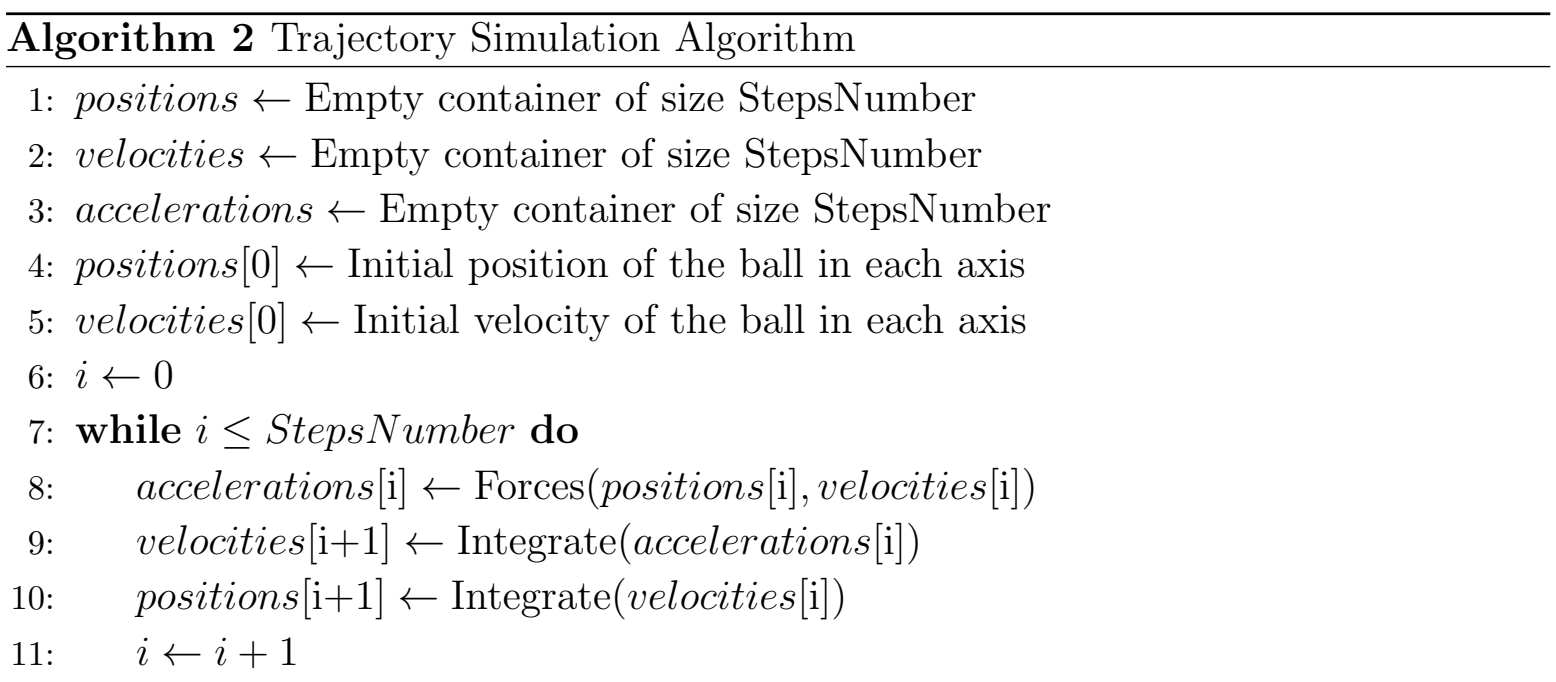

here, Forces refers to calculating and adding all forces acting on the ball (gravity, friction forces, spin forces in the rolling sphere model and the normal force of the surface, which depends on the surface shape), and obtain the acceleration applying Newton's Law. Both the shape of the trampoline and the model used affect the final trajectory, since the model determines how to calculate the value of the forces acting on the ball, and in particular uses the shape of the trampoline to adjust the normal force. As in the previous algorithm, Integrate refers to an Ordinary Differential Equation numerical solver, and the fourth order Runge-Kutta method was used as well.

Using this algorithm, we can find the best values for the unknown model parameters by simulating and optimizing how similar are the simulated trajectories to the observed ones, and then conclude which of the models is able to better describe the observed trajectories.

\subsection{Model comparison and optimization of unknown parameters}

5.5.1. Fixed trampoline optimization The first optimization over the unknown parameters of the models exposed in tables 3 and 4 aimed to do two comparisons:

(i) Comparison between the exponential trampoline (Equation 2) with $k$ and $c$ fitted to the real measures and the trampoline obtained using the trampoline shape model and Algorithm 1

(ii) Comparison between the point particle model and rolling sphere model

The four possible combinations were simulated, optimizing for all the unknown parameters, including $\sigma$ from Equation 2. For each of the four takes shown previously 
in Figure 4, the loss function minimized is the mean of the square euclidean distances between observed trajectories $x_{i}$ and simulated trajectories $\tilde{x}_{i}$ at each timestep $i$ :

$$
\mathcal{L}=\frac{1}{N} \sum_{i=0}^{N}\left\|x_{i}-\tilde{x}_{i}\right\|^{2}
$$

here, $\mathcal{L}$ is thought as a function of the values of the unknown parameters and its value is minimized using a search method combined with Nelder-Mead algorithm.

Both the trampoline obtained with the trampoline shape model and the exponentially fitted trampoline achieved similar performance in all the cases. The point particle model failed to determine parameters that could reproduce similar trajectories to the ones observed, but the rolling sphere model was able to take advantage of the initial spin parameter (which does not exist in the point particle model) to obtain a better fit to the four takes. As expected, this more complex model was able to better explain the observed trajectories. However, the spin value found in the optimal case was larger than the expected real one, probably to compensate mistakes of the model with respect to the real scenario. An example of performance of the rolling sphere model in the best fit case can be found in Figure 13. The Root Mean Square Error (RMSE, square root of the fitness function used) averaged over the takes achieved in this case is $0.09 \mathrm{~m}$.

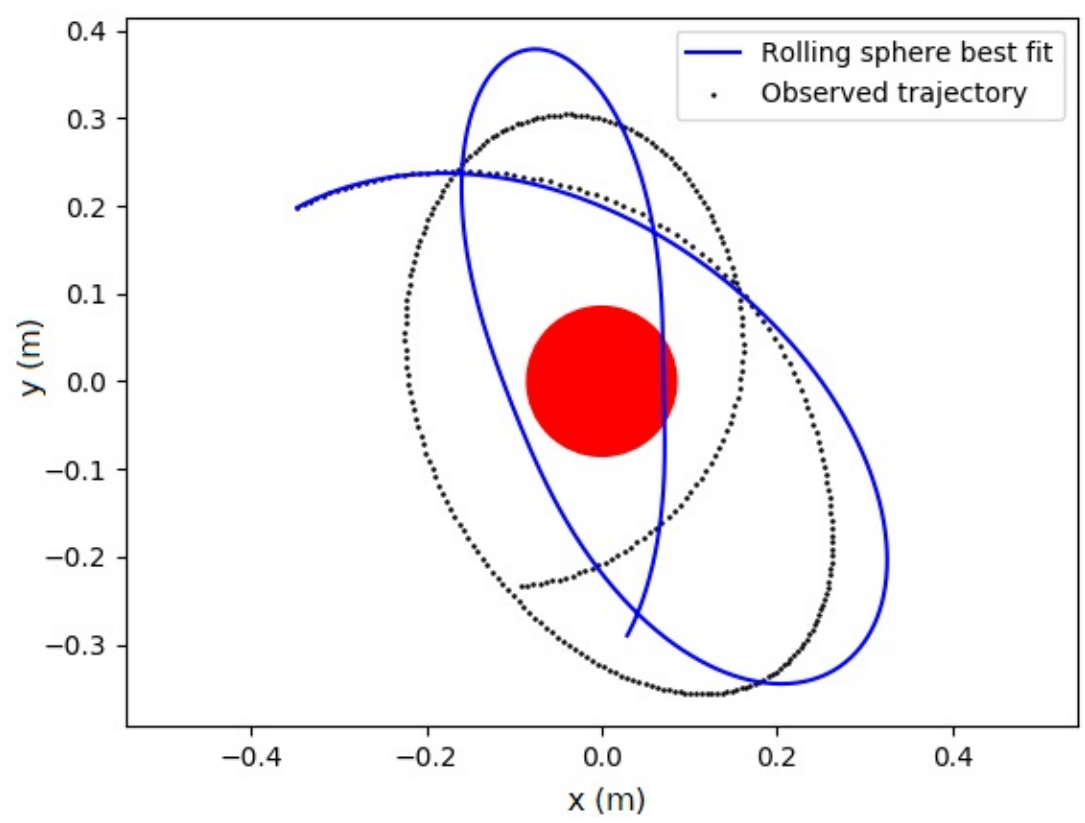

Figure 13: Example of a predicted trajectory of the rolling sphere model over the trampoline obtained using Algorithm 1, compared to the real observed trajectory

5.5.2. Free trampoline optimization Another optimization was performed, over the same fitness function, this time only with the exponential trampoline (not using the shape model trampoline) and letting $c$ and $k$ as parameters to be optimized, both in the 
case of the point model and the rolling sphere model. The results of both models can be seen in Figures 14 and 15. The RMSE achieved for each point is $0.05 \mathrm{~m}$ in the case of the rolling sphere model and $0.07 \mathrm{~m}$ in the case of the point model, a slight difference. However, the $c$ and $k$ parameters found while optimizing the point particle model are $0.25 \mathrm{~m}^{-1}$ and $0.83 \mathrm{~m}$, far from the real ones, while on the other hand, the parameters found by using the rolling sphere model are reasonably close to the observed ones at 2.4 $\mathrm{m}^{-1}$ and $0.43 \mathrm{~m}$, recalling that the observed ones were $1.8 \mathrm{~m}^{-1}$ and $0.44 \mathrm{~m}$.

Both facts lead to the conclusion that the rolling sphere model greatly outperforms the point particle model in explaining the experiment. The obtained results could even be improved by a model taking into account the possible sliding of the ball over the surface.

Point particle best fit
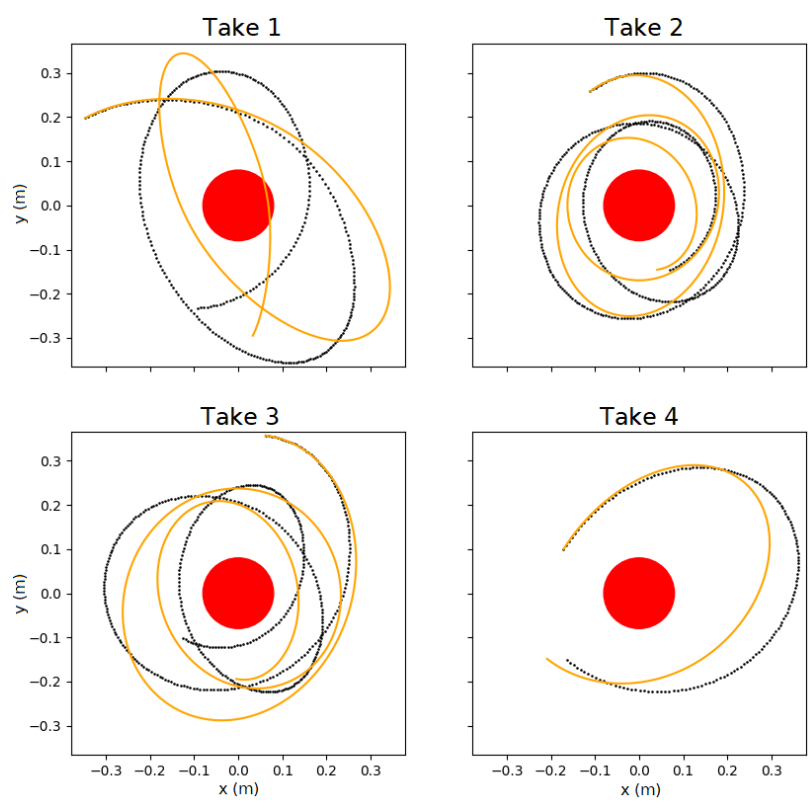

- Model fit

- Observed trajectory

Figure 14: Point particle model best fit trajectories after optimization (in orange), compared to the observed ones (in black) 
Rolling sphere model best fit

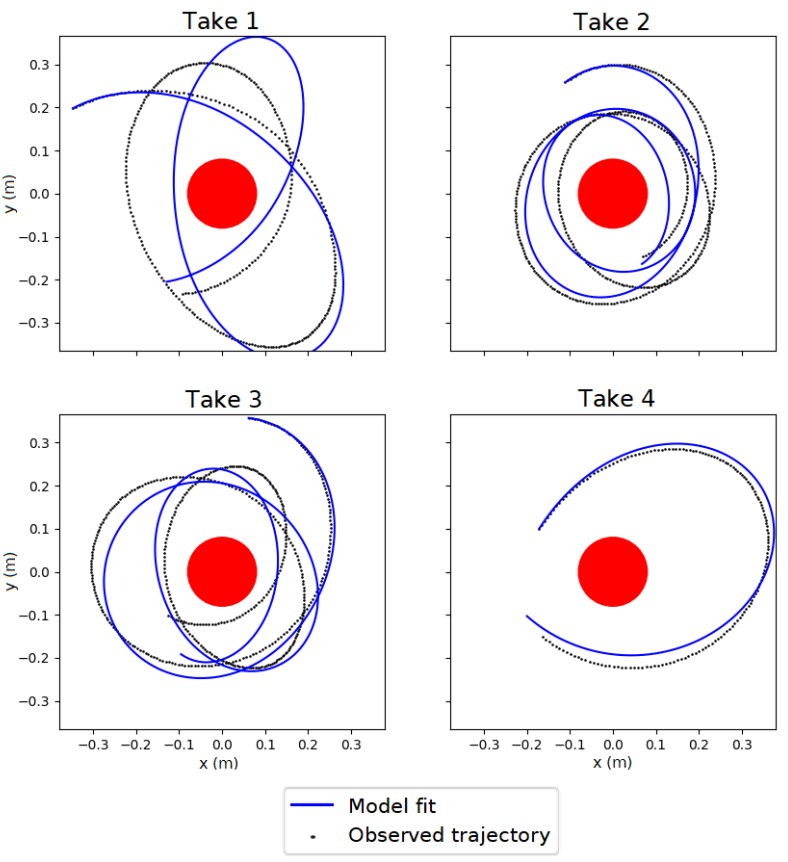

Figure 15: Rolling sphere model best fit trajectories after optimization (in blue), compared to the observed ones (in black)

\section{Conclusions}

The two main questions this article addressed were evaluating the rubber-sheet analogy and predicting the orbit of balls rolling on a warped trampoline, by using in both cases data collected from the experiment performed in Tibidabo park.

Firstly, no way of extending the analogy accurately beyond the rubber sheet being a visualization of the spacetime warping caused by masses has been found. It can not be considered as a complete spacetime where bodies follow geodesics, neither the orbits a ball follows on a warped trampoline are compatible with Newtonian and GR planetary orbits. The alternative model proposed by K.Thorne in [9], in which the rubber sheet only represents a slice of space instead of the full spacetime would help overcome some of the analogy deficits but would still not suffice to describe the apsidal precession observed in ball trajectories.

Regarding the second question, two different physical models were proposed and compared to describe the phenomenon. Computational simulations of both the trampoline and the ball movement concluded that the proposed rolling sphere model can predict accurately the experimental results, outperforming the other model proposed, the point particle model. 


\section{Acknowledgments}

We would like to thank the amusement park Tibidabo for offering their facilities and believing in FISIDABO project to promote youth talent in physics. We also want to thank Artur Paz for his support on the logistics of the experiment. We also want to extend our thanks to professor Francisco Marquès and PhD student Yago Herrera for their advice in the physical models. Finally we want to acknowledge the continuous efforts offered by Enginyeria Física from Universitat Politècnica de Catalunya.

\section{References}

[1] Ponnambalam M 2018 Emotional Component in Teaching and Learning The Physics Teacher 56 92-95

[2] Gupta A, Elby A and Danielak B A 2018 Exploring the entanglement of personal epistemologies and emotions in students thinking Phys. Rev. Phys. Educ. Res. 14010129

[3] FISIDABO webpage, fisidabo.upc.edu

[4] Buenda J J, Lopez H, Sanchis G and Pardo L C 2017 Modelling human behaviour in a bumper car ride using molecular dynamics tools: a student project European Journal of Physics 38035802

[5] Gurri P, Amat D, Espar J, Puig J, Gerard Jimenez G, Sendra L and Pardo L C 2017 Pendulum dynamics in an amusement park European Journal of Physics 38035005

[6] Tracker by Douglas Brown, found as open software in physlets.org/tracker/

[7] Pokorny P 2012 Geodesics Revisited Chaotic Modeling and Simulation (CMSIM) Jan 2012281298

[8] Middleton C A and Langston M 2014 Circular orbits on a warped spandex fabric American Journal of Physics $\mathbf{8 2}$ 287-294

[9] Thorne K 2003 Warping spacetime, published in The Future of Theoretical Physics and Cosmology: Celebrating Stephen Hawkings 60th Birthday. Available on www.ws5.com/spacetime/SpaceTime\%20Warp.pdf

[10] Carroll S M 1997 Lecture Notes on General Relativity. Available on arXiv:gr-qc/9712019.

[11] Kenneth Hong C M 2006 General Relativity: Warping of Space and Time. Article for GEK1508 Course; Einstein's Quantum Weirdness. 


\section{Annex}

In this annex are found the mathematical derivations of the equations used to implement the physical models in a computer program.

\subsection{Trampoline shape simulation}

The Tibidabo trampoline is a mesh of elastic cords, which can be approximately considered a continuous elastic surface. The Physical Models section presented a usual approach to simulate such surfaces: discretizing them as a grid of point masses joined by springs. In the end, the simulation is a mesh of connected springs, which resembles the original trampoline even more than the intended continuous surface. Crossings of the original elastic cords can be associated to the points of the grid and a segment of cord between crossings to a spring. This makes the transformation between the simulation and the real trampoline straight-forward.

Definitions The points in the grid are indexed by $(i, j)$, their positions are $\vec{r}_{i j}$. Following the mentioned association, the index range will be:

$$
0 \leq i \leq \text { Crossings }_{x-\text { axis }}+1 \quad 0 \leq j \leq \text { Crossings }_{y-\text { axis }}+1
$$

The extreme values of any of both indices correspond to boundary points that will be fixed in their place, at height 0 .

\begin{tabular}{|c|c|c|c|}
\hline Axis & Number of crossings & Size $(m)$ & $\rho_{\text {cord }}\left(\mathrm{kg} \cdot \mathrm{m}^{-1}\right)$ \\
\hline$x$ & 214 & 3.80 & \multirow{2}{*}{0.01} \\
\hline$y$ & 150 & 1.60 & \\
\hline
\end{tabular}

Table 5: Tibidabo trampoline characteristics. Except size, they are estimated

From the data in the Table 5 it is calculated the cord length between crossings in each axis, $a_{x}$ and $a_{y}$, when the trampoline is flat (not weight loaded):

$$
a_{x}=\frac{3.80}{215}=0.01767 m, \quad a_{y}=\frac{3.80}{151}=0.00744 m
$$

The simulation springs acquire that length when the spring mesh is flat and tensed, under the initial tension $T_{0}$, however the length of a non-deformed spring on the x-axis $l_{x}$ or $\mathrm{y}$-axis $l_{y}$ is unknown. To find them, consider the tension $T$ any spring following Hooke's Law generates when deformed by an amount $\Delta l$ :

$$
T=K \frac{\Delta l}{l} \Longrightarrow T_{0}=K \frac{a_{x}-l_{x}}{l_{x}}
$$




$$
\Longrightarrow \quad l_{x}=\frac{K}{K+T_{0}} a_{x}, \quad l_{y}=\frac{K}{K+T_{0}} a_{y} .
$$

Where $K$ is the Young's modulus of the springs, which should coincide with the Young's modulus of the cords. $K$ is chosen as parameter instead of the more common $k=\frac{K}{l}$ because it is an intrinsic property of the cord, not depending on the segment under study, whichever its length.

The last definition is that the total mass of the point $(i, j)$ is referred as $\Delta m_{i j}$ :

$$
\Delta m_{i j}=\left(l_{x}+l_{y}\right) \rho_{c o r d}+\frac{M}{n_{C}} \mathcal{I}_{C}(i, j)
$$

The first summand is meant to represent the mass of the elastic cords: four segments of cord come out of every crossing, as shown in Figure 16, and half of each segment contributes to the mass of the point. The second summand adds more mass to the points inside a circle $C$ in the center of the mesh of springs, to represent the bowling ball and the lifting weights. $\mathcal{I}_{C}$ is a function that indicates with a 1 or a 0 whether the point is in the circle $C . n_{C}$ is the total number of grid points inside $C$. The total mass loaded on the trampoline is $M=67.3 \mathrm{Kg}$.

Equations of motion To clarify the considered situation, the following figure shows a close view of one of the mobile grid points:

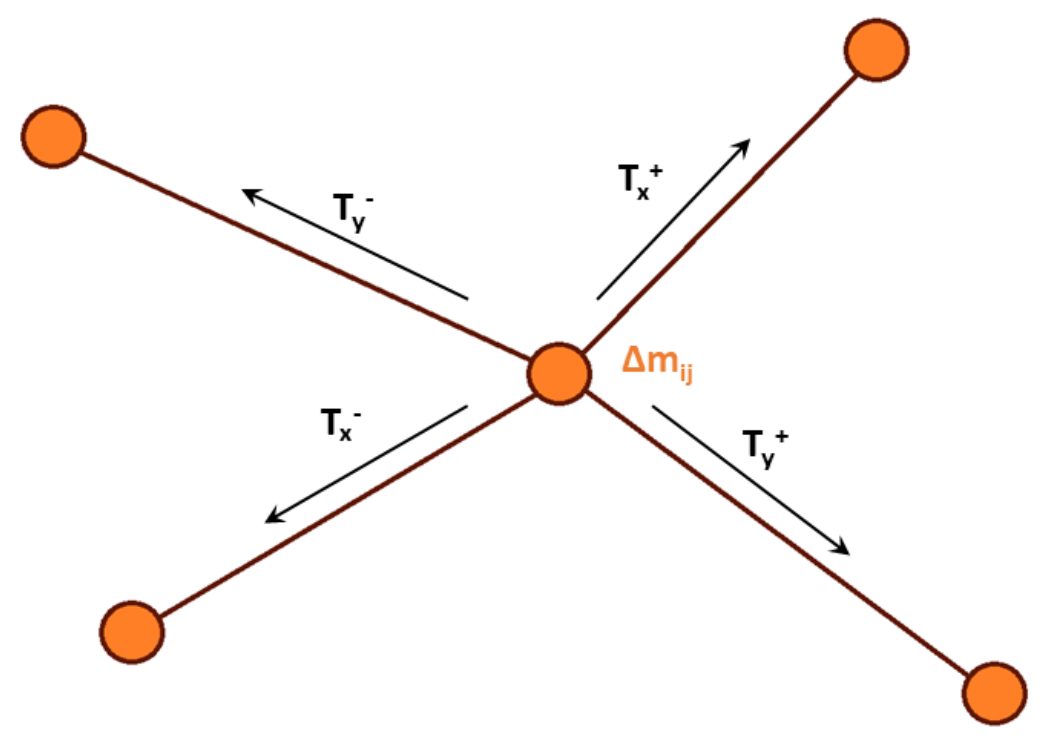

Figure 16: Point mass and its spring-linked neighbors

In the last figure, it is appreciable that any tension vector $\vec{T}$ is collinear to its spring. Adding up all the forces actuating on the point $(i, j)$ :

$$
\vec{F}_{(i, j)}=\vec{T}_{y(i, j)}^{-}+\vec{T}_{y(i, j)}^{+}+\vec{T}_{x(i, j)}^{-}+\vec{T}_{x(i, j)}^{+}-\Delta m_{i j} g \hat{z}
$$


The expression for one of the tension forces, $\vec{T}_{x}^{-}$(the one generated by the neighbour $(i-1, j))$ is developed:

$$
\begin{aligned}
& \vec{r}_{y(i, j)}^{-} \equiv \vec{r}_{i-1, j}-\vec{r}_{i, j} \\
& T_{y(i, j)}^{-}=K \frac{\left|\vec{r}_{y(i, j)}\right|-l_{y}}{l_{y}} \\
& \vec{T}_{y(i, j)}^{-}=T_{y(i, j)}^{-} \frac{\vec{r}_{y(i, j)}}{\left|\vec{r}_{y(i, j)}\right|}
\end{aligned}
$$

The calculation of the three other tension forces is analogous, only the corresponding indices need to be modified.

Finally, the equation of motion for each point $(i, j)$ is:

$$
\ddot{\vec{r}}_{i j}=\frac{\vec{F}_{(i, j)}}{\Delta m_{i j}}-\mu \dot{\vec{r}}_{i j}
$$

In which the added term, depending on the constant $\mu$, is the dissipative term that continuously reduces the mechanical energy of the system, forcing it to eventually halt in the desired equilibrium shape.

The mesh of springs and point masses will be initialized in a flat disposition, and when after a certain number of simulation steps the motions and accelerations come sufficiently close to zero the point masses disposition will be the estimation of the loaded Tibidabo's trampoline shape.

\subsection{Point particle model}

Total force $\vec{F}$ exerted on the punctual particle:

$$
\vec{F}=-m g \hat{z}+\vec{F}_{f}+\vec{F}_{N}
$$

The first term, $-m g \hat{z}$, is gravity, the second, $\vec{F}_{f}$, the dynamical friction force, and the last, $\vec{F}_{N}$, the normal force coming from the elastic trampoline. The particle can't cross the trampoline surface thanks to that normal force $\vec{F}_{N}$, which instead makes the particle stay over the surface.

Friction force is considered opposite to the particle velocity $\vec{v}$ and proportional to the normal force modulus (assumption (iv) of the point particle model):

$$
\vec{F}_{f}=-\mu F_{N} \hat{v}
$$

Where $\hat{v}$ indicates the unitary vector of $\vec{v}$ and $\mu$ is an unknown constant.

Normal force is calculated as follows: 
If the particle can only move inside the surface (assumption (ii)), then the velocity vector will have to be tangent to the surface, which is expressed as:

$$
\vec{v} \cdot \vec{n}=0 \quad \forall \vec{n} \in\left(T_{P} S\right)^{\perp}
$$

$P$ is the position of the particle, then $\vec{n}$ will be any vector normal to the surface (denoted by $S$ ) at the point $P$.

A normal vector at every point is found using the implicit form of the surface:

$$
\begin{aligned}
& F(x, y, z)=z-f(x, y)=0 \\
& \vec{n}=\vec{\nabla} F=\left[\begin{array}{lll}
-f_{x} & -f_{y} & 1
\end{array}\right]=\left[\begin{array}{ll}
-\vec{\nabla} f & 1
\end{array}\right]
\end{aligned}
$$

Where $f_{x}$ indicates the partial derivative of $f$ with respect to the $x$-axis. By always choosing this normal vector, it is possible to derivate 25 with respect to time:

$$
\vec{a} \cdot \vec{n}+\vec{v} \cdot \dot{\vec{n}}=0
$$

From $\vec{F}=m \vec{a}$ and since $\vec{F}_{f} \| \vec{v}$ and $\vec{F}_{N} \| \vec{n}$ (\| meaning parallel):

$$
\begin{aligned}
\vec{a} \cdot \vec{n} & =\left(-g \hat{z}+\frac{\vec{F}_{f}}{m}+\frac{\vec{F}_{N}}{m}\right) \cdot \vec{n} \\
& =-g+\frac{F_{N}}{m} n
\end{aligned}
$$

By denoting $H f$ the Hessian of $f$ :

$$
\begin{aligned}
\vec{v} \cdot \dot{\vec{n}} & =\left[\begin{array}{lll}
\dot{x} & \dot{y} & \dot{z}
\end{array}\right] \cdot\left(\left[\begin{array}{ll}
-\vec{\nabla} f & 1
\end{array}\right]\right) \\
& =\left[\begin{array}{lll}
\dot{x} & \dot{y} & \dot{z}
\end{array}\right] \cdot\left[-\left[\begin{array}{ll}
f_{x x} & f_{x y} \\
f_{y x} & f_{y y}
\end{array}\right]\left[\begin{array}{c}
\dot{x} \\
\dot{y}
\end{array}\right], 0\right] \\
& =-\left[\begin{array}{ll}
\dot{x} & \dot{y}
\end{array}\right] \operatorname{Hf}\left[\begin{array}{c}
\dot{x} \\
\dot{y}
\end{array}\right]
\end{aligned}
$$

Substituting on 27:

$$
\begin{aligned}
& -g+n \frac{F_{N}}{m}-\left[\begin{array}{ll}
\dot{x} & \dot{y}
\end{array}\right] H f\left[\begin{array}{l}
\dot{x} \\
\dot{y}
\end{array}\right]=0 \\
& F_{N}=\frac{m}{n}\left(g+\left[\begin{array}{ll}
\dot{x} & \dot{y}
\end{array}\right] H f\left[\begin{array}{l}
\dot{x} \\
\dot{y}
\end{array}\right]\right) \\
& \vec{F}_{N}=F_{N} \hat{n}=\frac{m}{(\vec{\nabla} f)^{2}+1}\left(g+\left[\begin{array}{ll}
\dot{x} & \dot{y}
\end{array}\right] H f\left[\begin{array}{c}
\dot{x} \\
\dot{y}
\end{array}\right]\right)\left[\begin{array}{ll}
-\vec{\nabla} f & 1
\end{array}\right]
\end{aligned}
$$

The resultant normal force $\vec{F}_{N}$ depends on the shape of the surface where the particle moves, specifically on $\vec{\nabla} f$ and $H f$. 
When the parametrization of the surface, $f(x, y)$, is known, gradient and the Hessian of the parametrized surface can be calculated. If the firstly proposed approximation of the trampoline surface is used: $f(x, y)=h(r(x, y)), h(r)=k e^{c r}, r(x, y)=$ $\sqrt{x^{2}+(\sigma y)^{2}}$ :

$$
\begin{gathered}
\vec{\nabla} f=\left[\begin{array}{l}
f_{x} \\
f_{y}
\end{array}\right]=h^{\prime}(r)\left[\begin{array}{l}
r_{x} \\
r_{y}
\end{array}\right]=h^{\prime}(r)\left[\begin{array}{c}
\frac{x}{r} \\
\frac{\sigma^{2} y}{r}
\end{array}\right] \\
H f=\left[\begin{array}{ll}
(\vec{\nabla} f)_{x} & (\vec{\nabla} f)_{y}
\end{array}\right] \\
=h^{\prime \prime}(r)\left[\begin{array}{l}
r_{x} \\
r_{y}
\end{array}\right]\left[\begin{array}{ll}
r_{x} & r_{y}
\end{array}\right]+h^{\prime}(r)\left[\begin{array}{cc}
r_{x x} & r_{x y} \\
r_{y x} & r_{y y}
\end{array}\right] \\
=h^{\prime \prime}(r)\left[\begin{array}{ll}
\left(\frac{x}{r}\right)^{2} & \frac{\sigma^{2} x y}{r^{2}} \\
\frac{\sigma^{2} x y}{r^{2}} & \left(\frac{\sigma^{2} y}{r}\right)^{2}
\end{array}\right]+h^{\prime}(r)\left[\begin{array}{cc}
\frac{1}{r}-\frac{x^{2}}{r^{3}} & \frac{\sigma^{2} x y}{r^{3}} \\
\frac{\sigma^{3}}{r}-\frac{\sigma^{4} y^{2}}{r^{3}}
\end{array}\right] \\
\left.h^{\prime \prime}(r)-\frac{h^{\prime}(r)}{r}\right) \vec{\nabla} r \vec{\nabla} r^{\top}+\frac{h^{\prime}(r)}{r}\left[\begin{array}{cc}
1 & 0 \\
0 & \sigma^{2}
\end{array}\right] \\
h^{\prime}(r)=c k e^{c r} \quad h^{\prime \prime}(r)=c^{2} k e^{c r}
\end{gathered}
$$

Equation (23) showed every force that actuates on the point particle. Afterwards, explicit expressions to calculate each force have been found: (24), (32). By applying Newton's Second Law the particle's acceleration $\vec{a}$ is obtained, then, its trajectory is predicted, given its initial conditions of position $\vec{x}(0)$ and velocity $\vec{v}(0)$ and using an integrator for $\vec{a}=\ddot{\vec{x}}$.

\subsection{Rolling sphere model}

The first step to build the equations of the rolling sphere model is to define $\vec{r}_{O}$ as the position of the sphere's center of mass and $\vec{r}_{P}$ as the position of the contact point between the surface and the sphere. The final objective is to obtain the mass center $O$ acceleration, $\ddot{\vec{r}}_{O}$. To accomplish it, all the constraints and relations of the physical model must be expressed mathematically.

If $\hat{n}$ is the unitary vector of $\vec{n}$, which always has a positive $z$ component and therefore points upwards, then the following relation holds:

$$
\vec{r}_{P}=\vec{r}_{O}+\overrightarrow{O P}, \quad \overrightarrow{O P}=-R \hat{n}
$$

Where $R$ is the radius of the sphere rolling over the surface. The relation can be easily checked looking at Figure 17. 


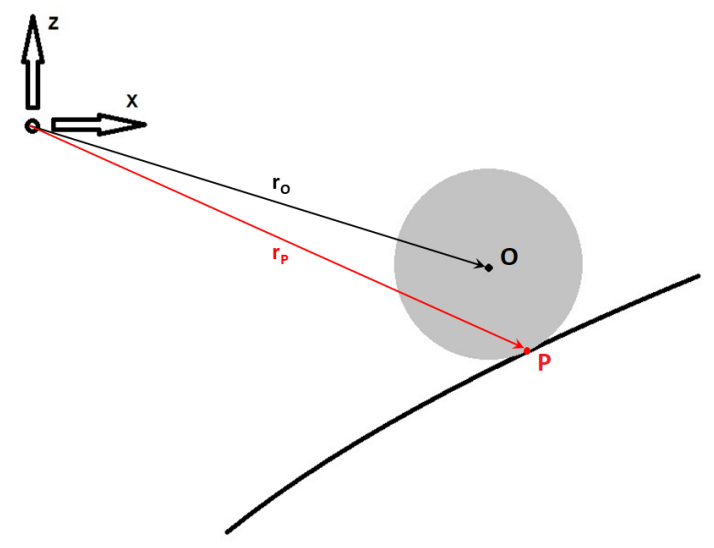

Figure 17: Diagram of the sphere rolling over the surface and its position vectors $\vec{r}_{O}$ and $\vec{r}_{P}$

Now, the model assumption (ii) can be rewritten as: the derivatives of the two position vectors, i.e. the center $O$ velocity and the contact point $P$ velocity, are parallel to the surface:

$$
\dot{\vec{r}}_{O} \cdot \vec{n}=0, \dot{\vec{r}}_{P} \cdot \vec{n}=0 \quad \forall \vec{n} \perp \text { Surface }
$$

The assumption (i), rolling without sliding, implies that the velocity of the sphere $\vec{v}_{S(P)}$ at the point of contact $P$ is zero $\left(\vec{v}_{S(P)}\right.$ does not have to be confused with the velocity of the contact point $\dot{\vec{r}}_{P}$, which is nonzero when the sphere moves). Then, rigid body kinematics tell us:

$$
\vec{v}_{O}+\vec{\omega} \times \overrightarrow{O P}=\vec{v}_{S(P)}=0
$$

Where $\vec{\omega}$ is the angular velocity vector and $\vec{v}_{O} \equiv \dot{\vec{r}}_{O}$.

The next equation to consider is Newton's Second Law, for simplicity the forces are split between the one exerted by the elastic trampoline on $P$ and gravity force:

$$
m \ddot{\vec{r}}=\vec{F}_{\text {trampoline }}-m g \hat{z}
$$

The following equation is the so called Newton's Second Law for rigid bodies, which takes a simple form due to our body being a sphere:

$$
\begin{aligned}
\vec{\tau} & =I \dot{\vec{\omega}} \\
\overrightarrow{O P} \times \vec{F}_{\text {tramp. }} & =\vec{\tau}
\end{aligned}
$$

Only the force exerted by the trampoline $\vec{F}_{\text {tramp }}$. contributes to the torque $\vec{\tau}$, because the gravity force acts on the center of mass. $I$ is the momentum of inertia, which on a solid sphere takes the value $I=\frac{2}{5} m R^{2}$. 
All relations have been considered, only algebraic manipulation is left to determine all the unknown variables $\left(\vec{F}_{\text {tramp. }}, \vec{\omega}, \ddot{\vec{r}}_{O}\right)$. To start, the variables are split into its normal and tangent components to the surface:

$$
\begin{aligned}
\vec{F}_{\text {tramp. }} & =\vec{F}_{N}+\vec{F}_{t} \\
\vec{\omega} & =\vec{\omega}_{s}+\vec{\omega}_{t} \\
\ddot{\vec{r}} & =\vec{a}_{n}+\vec{a}_{t}
\end{aligned}
$$

The $N$ or $n$ refers to normal, the $s$ refers to spin and $t$ to tangent component. To isolate the values of $\vec{\omega}_{t}$ and $\vec{F}_{t}$ from the equations 38 and 40 , the triple product identity will come in useful:

$$
\begin{aligned}
& \vec{a} \times(\vec{b} \times \vec{c})=\vec{b}(\vec{a} \cdot \vec{c})-\vec{c}(\vec{a} \cdot \vec{b}) \\
& \vec{\omega} \times \overrightarrow{O P}=-\dot{\vec{r}}_{O} \\
& \overrightarrow{O P} \times(\vec{\omega} \times \overrightarrow{O P})=-\overrightarrow{O P} \times \dot{\vec{r}}_{O} \\
& \vec{\omega}(\overrightarrow{O P} \cdot \overrightarrow{O P})-\overrightarrow{O P}(\overrightarrow{O P} \cdot \vec{\omega})=-\overrightarrow{O P} \times \dot{\vec{r}}_{O} \\
& \vec{\omega} R^{2}-R^{2}(\vec{\omega} \cdot \hat{n}) \hat{n}=R \hat{n} \times \dot{\vec{r}}_{O} \\
& R \vec{\omega}_{t}=\hat{n} \times \dot{\vec{r}}_{O} \\
& \overrightarrow{O P} \times \vec{F}_{\text {tramp. }}=I \dot{\vec{\omega}} \\
& \overrightarrow{O P} \times\left(\overrightarrow{O P} \times \vec{F}_{\text {tramp. }}\right)=\overrightarrow{O P} \times I \dot{\vec{\omega}} \\
& -R^{2} \vec{F}_{t}=I(\overrightarrow{O P} \times \dot{\vec{\omega}}) \\
& \vec{F}_{t}=\frac{2}{5} m(\dot{\vec{\omega}} \times \overrightarrow{O P})=\frac{2}{5} m R(\hat{n} \times \dot{\vec{\omega}})
\end{aligned}
$$

When the vectorial product is taken at both sides, a component of the vectors is lost, the one parallel to $\overrightarrow{O P}$. Therefore an scalar equation for each vectorial equation 38 and 40 was forgotten when the vectorial product was done. They can be recovered by scalarly multiplying both sides by a vector collinear to $\overrightarrow{O P}=-R \hat{n}$.

$$
\begin{aligned}
& \dot{\vec{r}}_{O} \cdot \hat{n}=\vec{\omega} \times \overrightarrow{O P} \cdot \hat{n}=0 \\
& I \dot{\vec{\omega}} \cdot \hat{n}=\overrightarrow{O P} \times \vec{F}_{\text {tramp. }} \cdot \hat{n}=0
\end{aligned}
$$

The first equation was already known, it is a reformulation of the condition of staying in contact with the trampoline 37, but the second one is new. They tell that both $\dot{\vec{r}}_{O}$ and $\dot{\vec{\omega}}$ don't have a normal component, they are tangent or parallel to the surface.

To know the value of $\vec{F}_{t}$ explicitly, the angular acceleration $\dot{\vec{\omega}}$ is needed. The tangent component $\vec{\omega}_{t}$ is already known, and its derivative is:

$$
\dot{\vec{\omega}}_{t}=\frac{d}{d t}\left(\frac{\hat{n} \times \dot{\vec{r}}_{O}}{R}\right)=\frac{\dot{\hat{n}} \times \dot{\vec{r}}_{O}}{R}+\frac{\hat{n} \times \ddot{\vec{r}}_{O}}{R}
$$


The normal component can not be explicitly known, but its derivative can still be calculated:

$$
\begin{aligned}
& \dot{\omega}_{s}=(\vec{\omega} \cdot \hat{n})^{\cdot}=\dot{\vec{\omega}} \cdot \hat{n}+\vec{\omega} \cdot \dot{\hat{n}}=\vec{\omega} \cdot \dot{\hat{n}} \\
& \dot{\vec{\omega}}_{s}=\left(\omega_{s} \hat{n}\right)^{\cdot}=\dot{\omega}_{s} \hat{n}+\omega_{s} \dot{\hat{n}}=(\vec{\omega} \cdot \dot{\hat{n}}) \hat{n}+\omega_{s} \dot{\hat{n}}
\end{aligned}
$$

It can be found that $\vec{\omega} \cdot \dot{\hat{n}}=\vec{\omega}_{t} \cdot \dot{\hat{n}}$, which requires less information to be known. This identity happens thanks to the fact that $\hat{n} \cdot \dot{\hat{n}}=0$ because the unitary vector $\hat{n}$ has a constant modulus. Now, it is possible to compute the value of $\vec{F}_{t}$ explicitly:

$$
\begin{aligned}
\vec{F}_{t} & =\frac{2}{5} m R(\hat{n} \times \dot{\vec{\omega}}) \\
\hat{n} \times \dot{\vec{\omega}} & =\hat{n} \times\left(\dot{\vec{\omega}}_{s}+\dot{\vec{\omega}}\right) \\
& =\hat{n} \times\left((\vec{\omega} \cdot \dot{\hat{n}}) \hat{n}+\omega_{s} \dot{\hat{n}}+\frac{\dot{\hat{n}} \times \dot{\vec{r}}_{O}}{R}+\frac{\hat{n} \times \ddot{\vec{r}}_{O}}{R}\right) \\
& =\hat{n} \times\left(\omega_{s} \dot{\hat{n}}+\frac{\dot{\hat{n}} \times \dot{\vec{r}}_{O}}{R}+\frac{\hat{n} \times \ddot{\vec{r}}_{O}}{R}\right)
\end{aligned}
$$

To simplify the previous expression it will be useful to use the tangent $\vec{a}_{t}$ component of the center's acceleration $\ddot{\vec{r}}_{O}$ :

$$
\begin{aligned}
\hat{n} \times\left(\hat{n} \times \ddot{\vec{r}}_{O}\right) & =-\vec{a}_{t} \\
\hat{n} \times\left(\dot{\hat{n}} \times \dot{\vec{r}}_{O}\right) & =\dot{\hat{n}}\left(\dot{\vec{r}}_{O} \cdot \hat{n}\right)-\dot{\vec{r}}_{O}(\hat{n} \cdot \dot{\hat{n}})=0 \\
\Longrightarrow \vec{F}_{T} & =\frac{2}{5} m R\left(\omega_{s} \hat{n} \times \dot{\hat{n}}-\frac{\vec{a}_{t}}{R}\right) \\
& =\frac{2}{5} m R \omega_{s} \hat{n} \times \dot{\hat{n}}-\frac{2}{5} m \vec{a}_{t}=: \vec{F}_{s}-\frac{2}{5} m \vec{a}_{t}
\end{aligned}
$$

Substituting in the 2nd Newton's Law (39) restricted to the tangent space:

$$
\begin{aligned}
m \vec{a}_{t} & =\vec{F}_{t}-(m g \hat{z})_{t} \\
& =\vec{F}_{s}-\frac{2}{5} m \vec{a}_{t}-m g(\hat{z})_{t} \\
& =\frac{5}{7}\left[\vec{F}_{s}-m g(\hat{z})_{t}\right], \quad(\hat{z})_{t}=\hat{z}-(\hat{z} \cdot \hat{n}) \hat{n}
\end{aligned}
$$

Thus, the net effect from the tangent force produced by the trampoline $\vec{F}_{t}$, the force that appears in order to change the rotation of the ball when necessary, is that all the tangent forces get their effect reduced by $5 / 7=\left(\frac{I}{m R^{2}}+1\right)^{-1}$ plus an extra force $\vec{F}_{s}=\frac{I}{R} \omega_{s} \hat{n} \times \dot{\hat{n}}$ which is proportional to the value of the spin $\omega_{s}$ and to how the surface is changing, $\hat{n} \times \dot{\hat{n}}$.

The normal component of the acceleration is calculated, equally to the point particle model, taking the derivative from the condition of moving inside the surface $\dot{\vec{r}}_{O} \cdot \hat{n}=0$ :

$$
\begin{aligned}
\ddot{\vec{r}}_{O} \cdot \hat{n}+\dot{\vec{r}}_{O} \cdot \dot{\hat{n}} & =0 \\
a_{n} & =-\dot{\vec{r}}_{O} \cdot \dot{\hat{n}}
\end{aligned}
$$


Now, the acceleration of the center $O$ is expressed as a function of $\omega_{s}, \hat{n}$ and $\dot{\hat{n}}$ :

$$
\ddot{\vec{r}}_{O}=-\left(\dot{\vec{r}}_{O} \cdot \dot{\hat{n}}\right) \hat{n}+\frac{2}{7} R \omega_{s} \hat{n} \times \dot{\hat{n}}-\frac{5}{7} g(\hat{z}-(\hat{z} \cdot \hat{n}) \hat{n})
$$

The value of $\omega_{s}$ can be numerically integrated from its derivative, which only depends on $\vec{\omega}_{t}$ and $\dot{\hat{n}}$ :

$$
\begin{aligned}
\dot{\omega}_{s} & =\vec{\omega}_{t} \cdot \dot{\hat{n}}=\frac{\hat{n} \times \dot{\vec{r}}_{O}}{R} \cdot \dot{\hat{n}} \\
R \dot{\omega}_{s} & =-\dot{\vec{r}}_{O} \cdot(\hat{n} \times \dot{\hat{n}})
\end{aligned}
$$

However, to continue a problem is found: $\hat{n}$ and $\dot{\hat{n}}$ depend on $\vec{r}_{P}$ and $\dot{\vec{r}}_{P}$, but only $\vec{r}_{O}$ and $\dot{\vec{r}}_{O}$ can be obtained from $\ddot{\vec{r}}_{O}$ integration. Derivative of equation 36 allows obtaining $\dot{\vec{r}}_{P}$ from $\dot{\vec{r}}_{O}$ as the solution of a matrix linear system, (as $\dot{\hat{n}}$ depends linearly of $\dot{\vec{r}}_{P}$ ):

$$
\dot{\vec{r}}_{P}+R \dot{\hat{n}}\left(\vec{r}_{P}, \dot{\vec{r}}_{P}\right)=\dot{\vec{r}}_{O}
$$

Then, $\vec{r}_{P}$ can be obtained from $\dot{\vec{r}}_{P}$ through integration and finally the problem is solved since all the required values to calculate $\ddot{\vec{r}}_{O}$ are known. Before concluding, to solve the system of linear equations it was needed to explicitly know them:

$$
\begin{aligned}
\dot{\hat{n}} & =\frac{d}{d t}\left(\frac{\vec{n}}{n}\right)=\frac{\dot{\vec{n}}}{n}-\frac{\vec{n}}{n^{2}} \dot{n} \\
0=\dot{\hat{n}} \cdot \vec{n} & =\frac{\dot{\vec{n}} \cdot \vec{n}}{n}-\dot{n} \Longrightarrow \dot{n} n=\dot{\vec{n}} \cdot \vec{n} \\
\dot{\hat{n}} & =\frac{\dot{\vec{n}}}{n}-\dot{\vec{n}} \cdot \vec{n} \frac{\vec{n}}{n^{3}}=\frac{(\dot{\vec{n}})_{t}}{n}=\left(\frac{I d-\hat{n} \cdot \hat{n}^{\top}}{n}\right) \dot{\vec{n}}
\end{aligned}
$$

The derivative of the normal vector, $\dot{\vec{n}}$, can be recovered from calculations in the point particle model part of the annex:

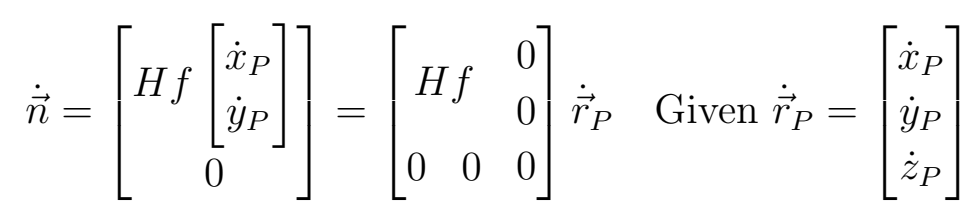

The linear system becomes explicitly:

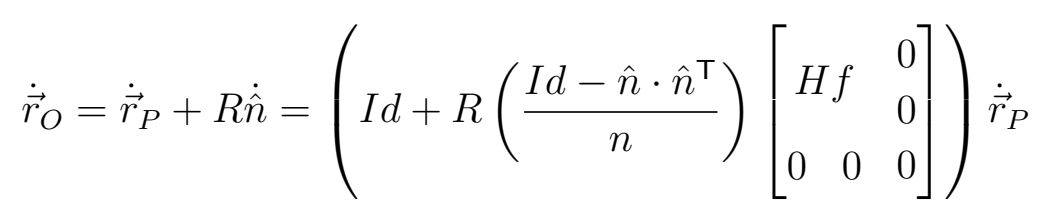

Although it has not been still needed, the value of the normal force exerted by the trampoline $\vec{F}_{N}$ can be calculated and it has exactly the same value as in the point particle model:

$$
\begin{aligned}
m \ddot{\vec{r}}_{O} \cdot \hat{n} & =\left(\vec{F}_{\text {tramp. }}-m g \hat{z}\right) \cdot \hat{n} \\
m a_{n} & =F_{N}-m g \hat{z} \cdot \hat{n} \\
\vec{F}_{N} & =m\left(\frac{g \hat{z} \cdot \vec{n}}{n}+a_{n}\right) \hat{n}=m\left(\frac{g}{n}-\dot{\vec{r}}_{O} \cdot \dot{\hat{n}}\right) \hat{n}
\end{aligned}
$$


Extra Considerations On the developed model, the friction force between the trampoline and the ball has been ignored. If the ball rolled ideally, the friction with the trampoline surface would be zero, as they are stationary one to the other at the point of contact P. However, the rolling is not ideal and the contact between the surface and the sphere is not a point, the contact area is broader because the trampoline deforms to accommodate the ball. This broader contact allows a friction force or rolling resistance to appear, this force doesn't generate torque and as a simple approximation is opposed to velocity and proportional to the normal force modulus $\vec{F}_{f}=-C_{r r} F_{N} \hat{v}$, equally as in the point particle model.

Another friction force that wasn't considered during the development of the model and is added a posteriori is the friction force on the spin $\omega_{s}$, which makes a spinning ball stop. It is also caused due to not being punctual the contact zone between the sphere and the bed. This friction can be implemented adding a term to the derivative $\dot{\omega}_{s}$, that will be proportional to $F_{N}$ and oppose to the spin:

$$
R \dot{\omega}_{s}=-\dot{\vec{r}}_{O} \cdot(\hat{n} \times \dot{\hat{n}})-\operatorname{sign}\left(\omega_{s}\right) \eta F_{N}
$$

This spin friction is not considered to generate a net force on the center of masses of the ball and only creates a torque on the ball in the $\hat{n}$ direction. Therefore, it doesn't interfere with the torque generated by the tangent force of the trampoline $\overrightarrow{O P} \times \vec{F}_{t}$ which is orthogonal to $\hat{n}$. It is clarifying to note that the value of the tangent torque $\vec{\tau}_{t}=\vec{\tau}-(\vec{\tau} \cdot \hat{n}) \hat{n}=\overrightarrow{O P} \times \vec{F}_{t}$ is determined completely by $(\dot{\vec{\omega}})_{t}$ or equivalently by: the imposition that the sphere rolls without sliding and the value of $\omega_{s} \cdot \dot{\hat{n}}$. Then, adding this spin friction ad hoc doesn't change the already deduced value of $\vec{F}_{t}$.

Moreover, air could also create friction against the movement of the ball. That friction would not depend on the normal force and instead be proportional or quadratic with respect to velocity. This type of friction is not considered in this model.

Concluding, the ordinary differential equation (ODE) system that should be solved is the one expressed in the Physical Models section, in equation 9 and its subsequent equations. The parameters on which the ODE depends are listed on Table 4 of the same section. 